\title{
Existence of global attractor for one-dimensional weakly damped nonlinear Schrödinger equation with Dirac interaction and artificial boundary condition in half-line
}

\author{
Mostafa Abounouh, Hassan Al Moatassime and Abderrazak Chrif*
}

\section{"Correspondence:}

abderrazak.chrifi@gmail.com Department of Mathematics,

Faculty of Science and Technology, Cadi Ayyad University, B.P. 549,

Av. Abdelkarim Elkhattabi, Guéliz,

Marrakesh, 40000, Morocco

\section{Springer}

\begin{abstract}
We consider a nonlinear Schrödinger equation with Dirac interaction defect. Moreover, non-standard boundary conditions are introduced in connection to the behavior of the solutions. First, we prove that this kind of Schrödinger equation can be characterized by an autonomous dynamical system. Then, based on this result, we show that such an equation possesses a maximal compact attractor in the weak topology of $\mathbf{H}^{1}$
\end{abstract}

Keywords: damped nonlinear Schrödinger equation; Dirac interaction defect; artificial boundary condition; autonomous dynamical system; global attractor

\section{Introduction}

In this paper, we consider a nonlinear Schrödinger equation (NLS) with Dirac interaction. Such interaction is introduced in order to model physically the presence of impurity (see, for instance, [1-4]).

The NLS equation under study is described by

$$
i u_{t}+\frac{1}{2} u_{x x}+q \delta_{a} u+g\left(|u|^{2}\right) u+i \gamma u=f \quad \text { in } \boldsymbol{\Omega} \times \mathbb{R}_{+},
$$

with $\boldsymbol{\Omega} \subset \mathbb{R}$ and the unknown solution $u=u(x, t)$ maps $\boldsymbol{\Omega} \times \mathbb{R}_{+}$into $\mathbb{C}$. $\delta_{a}$ is the Dirac distribution which describes a defect at point $a \in \boldsymbol{\Omega}$. The parameter $q \in \mathbb{R}$ represents its intensity (see [3, 4] for more details). The effect of the damping parameter $\gamma>0$ is introduced in order to study the asymptotic behavior of the solution to the Schrödinger equation (1). The external time-independent forcing $f \in \mathbf{L}^{2}(\boldsymbol{\Omega})$ is added to avoid the triviality feature of the dynamic. The functional term $g$ is introduced in order to provide a generalization of the classical nonlinear NLS model.

The model (1) arises in many applications, quantum mechanics, hydrodynamic, optic, see for an overview [5-10]. In the case when $a=\gamma=0, g(s)=s$ and $f=0$, this introduced model coincides with the model $[1,2]$. Note that in the literature there are few studies for the case $q \neq 0$. A variety of physical models involve this case, see, for example, $[3,11,12]$ and the references therein.

(c) The Author(s) 2017. This article is distributed under the terms of the Creative Commons Attribution 4.0 International License (http://creativecommons.org/licenses/by/4.0/), which permits unrestricted use, distribution, and reproduction in any medium, provided you give appropriate credit to the original author(s) and the source, provide a link to the Creative Commons license, and indicate if changes were made. 
The long-time solutions behavior of nonlinear equation (1) has been already studied in the literature. In the case when $q=0$, with the assumption that $\boldsymbol{\Omega}$ is bounded, the existence of a finite dimensional maximal attractor in $\mathbf{H}^{\mathbf{1}}(\boldsymbol{\Omega})$ was proved in [13]. In [14], it was shown that the long-time behavior is described in terms of the existence of a global attractor belonging to $\mathbf{H}^{\mathbf{1}}(\boldsymbol{\Omega})$ with $\boldsymbol{\Omega}=\mathbb{R}^{n}$ and $n \leq 3$. For bounded $\boldsymbol{\Omega} \subset \mathbb{R}$ and with periodic boundary conditions, the smoothness of the global attractor in $\mathbf{H}^{2}(\boldsymbol{\Omega})$ was proved in [15]. In turn, the case $q \neq 0$ was studied in [3] in which it was shown that equation (1) is well posed in $\mathbf{H}^{\mathbf{1}}(\mathbb{R})$ for a simple particular potential function $g(s)=s$. In this simple case, it proved in [16] that the long-time behavior can be described by the existence of a global attractor in $\mathbf{H}^{\mathbf{1}}(\mathbb{R})$.

With regard to these previous works, the aim of this paper is to investigate the nonlinear Schrödinger (NLS) equation (1) in the case $\boldsymbol{\Omega}=$ ] $-\infty, \mathbf{0}$ [ with Dirac interaction defect. In addition, we consider artificial boundary conditions at the limit point 0 in order to avoid the perturbations on the behavior of the solutions caused by the boundary conditions. This issue was treated in [17-19] in connection to the numerical behavior of the solutions. Our main results that are stated and proved are as follows:

(i) The Schrödinger equation can be characterized by a dissipative autonomous dynamical system;

(ii) Existence of a maximal compact attractor in the weak topology of $\mathbf{H}^{\mathbf{1}}(\boldsymbol{\Omega})$.

In fact, based on our first result (i), by using dissipativity consideration and a splitting technique, we show that the Schrödinger equation possesses a maximal compact attractor in the weak topology of $\mathbf{H}^{\mathbf{1}}(\boldsymbol{\Omega})$.

The remainder of the paper is organized as follows. In Section 2, we provide some preliminaries and necessary key technical results. Section 3 introduces an autonomous dynamical system in order to study the asymptotic behavior of the solutions to the Schrödinger equation. Section 4 deals with the dissipativity issue of the introduced dynamical autonomous system for the characterization of an absorbing bounded set. In Section 5 we present a splitting technical approach to study the asymptotic compactness of the semigroup associated with the introduced autonomous dynamical system. In Section 6, we prove the precompactness of the attractor in the weak topology of $\mathbf{H}^{\mathbf{1}}(\boldsymbol{\Omega})$. Finally, Section 7 gives some concluding remarks.

\section{Problem formulation and preliminaries}

Throughout this paper, we use the following notations.

For a subset $\boldsymbol{\Omega}$ of $\mathbb{R}$ and $p \in \overline{\mathbb{R}}_{+}, L^{p}(\Omega)$ (the Lebesgue space of measurable function $u$ : $\boldsymbol{\Omega} \rightarrow \mathbb{C}$ ) is denoted by

$$
\begin{aligned}
& \mathbf{L}^{\mathbf{p}}(\boldsymbol{\Omega})=\left\{\left.u\left|\int_{\boldsymbol{\Omega}}\right| \mathbf{u}(\mathbf{x})\right|^{\mathbf{p}} \mathbf{d x}<+\infty\right\}, \\
& \mathbf{L}^{\infty}(\boldsymbol{\Omega})=\{\mathbf{u} \mid \exists \mathbf{M}>\mathbf{0} \text { such that }|\mathbf{u}(\mathbf{x})|<\mathbf{M} \text { p.p on } \boldsymbol{\Omega}\} .
\end{aligned}
$$

These spaces are endowed respectively with the norms $\|u\|_{L^{p}(\Omega)}=\left(\int_{\Omega}|u(x)|^{p} d x\right)^{\frac{1}{p}}$ and $\|u\|_{L^{\infty}(\Omega)}=\inf _{M>0}\{M|| u(x) \mid<M$, p.p on $\boldsymbol{\Omega}\}$. The set $\mathbf{H}^{\mathbf{1}}(\boldsymbol{\Omega})$ denotes the Sobolev space defined by $\mathbf{H}^{\mathbf{1}}(\boldsymbol{\Omega})=\left\{\mathbf{u} \in \mathbf{L}^{2}(\boldsymbol{\Omega}) ; \mathbf{u}_{\mathbf{x}} \in \mathbf{L}^{2}(\boldsymbol{\Omega})\right\}$, where the derivation $u_{x}$ is taken in the distribution sense. This space is endowed with the norm $\|u\|_{H^{1}(\Omega)}=\left(\|u\|_{L^{2}(\Omega)}^{2}+\left\|u_{x}\right\|_{L^{2}(\Omega)}^{2}\right)^{\frac{1}{2}}$. 
Now, we introduce the nonlinear Schrödinger equation (NLS), and we discuss the relevant issue of its boundary conditions as well as its extended domain $\boldsymbol{\Omega}=]-\infty, \mathbf{0}$ [.

Let $\delta_{a}$ be Dirac interaction defect at point $a$, then our NLS equation under study is given by

$$
i u_{t}+\frac{1}{2} u_{x x}+q \delta_{a} u+g\left(|u|^{2}\right) u+i \gamma u=f \quad \text { in } \boldsymbol{\Omega} \times \mathbb{R}_{+},
$$

where $\boldsymbol{\Omega}=]-\infty, \mathbf{0}\left[, a<0, f\right.$ belongs to $\mathbf{L}^{\mathbf{2}}(\boldsymbol{\Omega})$. The functional $g \in \mathbf{C}^{\mathbf{1}}([\mathbf{0},+\infty[, \mathbb{R})$ is a smooth function such that there exist constants $C \geq 0, \alpha_{1}>0, \alpha_{2}>0$ and $\theta \in[0,2$ [ satisfying

$$
\left\{\begin{array}{l}
\left|g^{\prime}(r)\right| \leq C \quad \text { for } r \geq 0, \\
g(r) \leq \alpha_{1}\left(1+r^{\theta}\right) \quad \text { for } r \geq 0, \\
G(r)=\int_{0}^{r} g(s) d s \quad \text { with }|G(r)| \leq \alpha_{2} r\left(1+r^{\theta}\right) \text { for } r \geq 0 .
\end{array}\right.
$$

We associate with NLS equation (2) artificial boundary conditions on $x=0$ for $t \geq 0$ in order to avoid the perturbation effect on the behavior of the solutions resulting from the reflection at the boundary $\{0\}$. This important issue was firstly considered in [17-19]. Such boundary conditions are given by

$$
\partial_{n} u(0, t)+\sqrt{2} e^{-i \pi / 4} e^{-\gamma t} e^{i \mathbb{V}(0, t)} \partial_{t}^{1 / 2}\left(e^{\gamma t} e^{-i \mathbb{V}(0, t)} u(0, t)\right)=0 \quad \text { for } t \in \mathbb{R}_{+},
$$

where $\partial_{n}$ represents the normal derivative operator. $\mathbb{V}$ is the phase function defined by $\mathbb{V}(x, t)=\int_{0}^{t} g\left(|u(x, s)|^{2}\right) d s$. The operator $\partial_{t}^{1 / 2}$ represents the Riemann-Liouville fractional derivative of $\frac{1}{2}$ order defined by

$$
\partial_{t}^{1 / 2}(h(t))=\frac{1}{\sqrt{\pi}} \partial_{t}\left(\int_{-\infty}^{t} \frac{h(s)}{\sqrt{t-s}} d s\right) .
$$

The initial data $u_{0} \in \mathbf{H}^{\mathbf{1}}(\boldsymbol{\Omega})$ is given at $t=0$, and we shall suppose that its support is in $\boldsymbol{\Omega}$

$$
u(x, 0)=u_{0}(x) \quad \text { for } x \in \mathbf{\Omega} .
$$

For the extended domain $\boldsymbol{\Omega}=$ ] $-\infty, \mathbf{0}$, the existence of the solutions to NLS equation (2)-(4)-(6) ant its well-posedness can be found in [20, 21]. In what follows, we provide a sketch of the proof of this result.

Theorem 1 Under the previous assumption, there exists a unique function $u \in \mathbf{C}^{\mathbf{0}}([\mathbf{0}, \mathbf{T}]$; $\left.\mathbf{H}^{\mathbf{1}}(\boldsymbol{\Omega})\right) \cap \mathbf{C}^{\mathbf{1}}\left([\mathbf{0}, \mathbf{T}] ;\left[\mathbf{H}^{\mathbf{1}}(\boldsymbol{\Omega})\right]^{\prime}\right)$ solution to NLS equation equation (2)-(4)-(6), where $\mathbf{C}^{\mathbf{k}}\left([\mathbf{0}, \mathbf{T}] ; \mathbf{H}^{\mathbf{1}}(\boldsymbol{\Omega})\right)$ is the space of $k$ times continuously differentiable functions on $[0, T]$ in $H^{1}(\Omega)$ and $\left[\mathbf{H}^{\mathbf{1}}(\boldsymbol{\Omega})\right]^{\prime}$ is the dual of $\mathbf{H}^{\mathbf{1}}(\boldsymbol{\Omega})$.

Sketch of the proof This proof is divided into three steps based on the Faedo-Galerkin method.

In the first step, by projection on the finite dimensional orthonormal basis of $\mathbf{H}^{\mathbf{1}}(\boldsymbol{\Omega})$, we construct a sequence of approximate equations, each of these equations possesses a 
solution via the Cauchy-Lipschitz criteria. Hence, we have constructed a sequence of approximate solutions.

The second step consists of obtaining estimation conditions to legitimate the convergence of approximate solutions to a limit. The third step shows that this limit is exactly a solution to NLS equation. Finally, we prove that this limit is unique and provides the global solution to NLS equation with regard to (2)-(4)-(6).

In our forthcoming analysis, we will use a slightly modified lemma of Lemma 2.1 in [22]. For this purpose we recall that for $s \in \mathbb{R}$, the Sobolev space with non-integer order $s$ is defined by

$$
\mathbf{H}^{\mathrm{s}}(\mathbb{R}):=\left\{\mathbf{u} \in \mathbf{L}^{2}(\mathbb{R}): \mathcal{F}^{-1}\left[\left(\mathbf{1}+|\xi|^{2}\right)^{\frac{\mathrm{s}}{2}} \mathcal{F} \mathbf{u}\right] \in \mathbf{L}^{2}(\mathbb{R})\right\}
$$

where $\mathcal{F}$ is the Fourier transform. The Sobolev space $\mathbf{H}^{\mathbf{s}}(\mathbf{0}, \mathbf{T})$ represents the set of restrictions of functions from $\mathbf{H}^{\mathbf{s}}(\mathbb{R})$ to $[\mathbf{0}, \mathbf{T}]$.

Lemma 1 Let $\phi \in \mathbf{H}^{\mathbf{1} / \mathbf{4}}(\mathbf{0}, \mathbf{T})$ and $\psi \in \mathbf{H}^{\mathbf{3} / \mathbf{4}}(\mathbf{0}, \mathbf{T})$ with $\psi(0)=0$ be two functions extended by zero beyond $T$, that is, for all $s>T$ and $s<0$. Then the following inequalities hold true:

(i) $\operatorname{Re}\left(e^{ \pm i \pi / 4} \int_{0}^{+\infty} e^{-\gamma t} \bar{\phi} \partial_{t}^{1 / 2} \phi d t\right) \geq 0$,

(ii) $\operatorname{Re}\left(e^{-i \pi / 4} \int_{0}^{+\infty} e^{-\gamma t} \overline{\psi_{t}} \partial_{t}^{1 / 2} \psi d t\right) \geq 0$,

where $\gamma>0$.

Proof Inequality (i): We apply the Plancherel identity to $e^{-\frac{\gamma t}{2}} \phi$ and $e^{-\frac{\gamma t}{2}} \partial_{t}^{1 / 2} \phi$ using $\mathcal{L}\left(e^{-\frac{\gamma t}{2}} \phi\right)(s)=\mathcal{L}(\phi)\left(s+\frac{\gamma}{2}\right)$ and $\mathcal{L}\left(\partial_{t}^{1 / 2} \phi\right)(s)=\sqrt{s} \mathcal{L}(\phi)(s)$, where the operator $\mathcal{L}$ is the Laplace transform. Then we have

$$
\begin{aligned}
\int_{0}^{+\infty} e^{-\gamma t} \bar{\phi} \partial_{t}^{1 / 2} \phi d t & =\frac{1}{2 \pi} \int_{-\infty}^{+\infty} \overline{\mathcal{L}\left(e^{-\frac{\gamma t}{2}} \phi\right)(i v)} \mathcal{L}\left(e^{-\frac{\gamma t}{2}} \partial_{t}^{1 / 2} \phi\right)(i v) d v \\
& =\frac{1}{2 \pi} \int_{-\infty}^{+\infty} \overline{\mathcal{L}(\phi)\left(i v+\frac{\gamma}{2}\right)} \mathcal{L}\left(\partial_{t}^{1 / 2} \phi\right)\left(i v+\frac{\gamma}{2}\right) d v \\
& =\frac{1}{2 \pi} \int_{-\infty}^{+\infty} \sqrt{\frac{\gamma}{2}+i v \mid}\left|\mathcal{L}(\phi)\left(i v+\frac{\gamma}{2}\right)\right|^{2} d v
\end{aligned}
$$

As $\gamma>0$ and $v \in \mathbb{R}$, we have

$$
\frac{\gamma}{2}+i v=\sqrt{\frac{\gamma^{2}}{4}+v^{2}} e^{i \theta} \quad \text { with } \theta \in\left[-\frac{\pi}{2} ; \frac{\pi}{2}\right]
$$

which gives

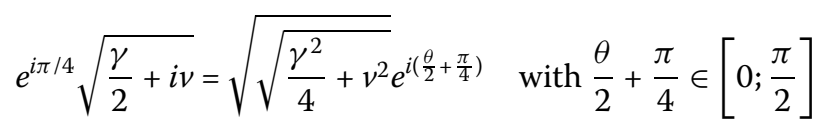


Abounouh et al. Advances in Difference Equations ( 2017) 2017:137

Page 5 of 24

and

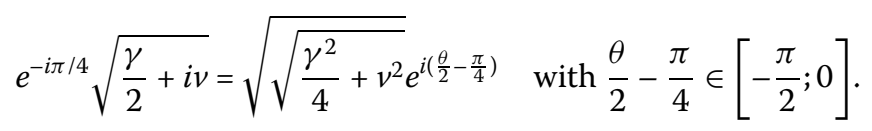

Since we have

$$
\operatorname{Re}\left(e^{i \pi / 4} \sqrt{\frac{\gamma}{2}+i v}\right) \geq 0, \quad \operatorname{Re}\left(e^{-i \pi / 4} \sqrt{\frac{\gamma}{2}+i \nu}\right) \geq 0,
$$

and by using the equalities

$$
e^{i \pi / 4} \int_{0}^{+\infty} e^{-\gamma t} \bar{\phi} \partial_{t}^{1 / 2} \phi d t=\frac{e^{i \pi / 4}}{2 \pi} \int_{-\infty}^{+\infty} \sqrt{\frac{\gamma}{2}+i v}\left|\mathcal{L}(\phi)\left(i v+\frac{\gamma}{2}\right)\right|^{2} d v
$$

and

$$
e^{-i \pi / 4} \int_{0}^{+\infty} e^{-\gamma t} \bar{\phi} \partial_{t}^{1 / 2} \phi d t=\frac{e^{-i \pi / 4}}{2 \pi} \int_{-\infty}^{+\infty} \sqrt{\frac{\gamma}{2}+i v}\left|\mathcal{L}(\phi)\left(i v+\frac{\gamma}{2}\right)\right|^{2} d \nu
$$

we can deduce inequality (i).

Inequality (ii): Since $\psi(0)=0$, we have

$$
\begin{aligned}
\int_{0}^{+\infty} e^{-\gamma t} \overline{\psi_{t}} \partial_{t}^{1 / 2} \psi d t & =\frac{1}{2 \pi} \int_{-\infty}^{+\infty} \overline{\mathcal{L}\left(e^{-\frac{\gamma t}{2}} \psi_{t}\right)(i v)} \mathcal{L}\left(e^{-\frac{\gamma t}{2}} \partial_{t}^{1 / 2} \psi\right)(i v) d v \\
& =\frac{1}{2 \pi} \int_{-\infty}^{+\infty} \overline{\mathcal{L}\left(\psi_{t}\right)\left(i v+\frac{\gamma}{2}\right)} \mathcal{L}\left(\partial_{t}^{1 / 2} \psi\right)\left(i v+\frac{\gamma}{2}\right) d v \\
& =\frac{1}{2 \pi} \int_{-\infty}^{+\infty}\left(\frac{\gamma}{2}-i v\right) \sqrt{\frac{\gamma}{2}+i v}\left|\mathcal{L}(\psi)\left(i v+\frac{\gamma}{2}\right)\right|^{2} d \nu
\end{aligned}
$$

As $\gamma \geq 0$ and $\nu \in \mathbb{R}$, we have

$$
\frac{\gamma}{2}+i v=\sqrt{\frac{\gamma^{2}}{4}+v^{2}} e^{i \theta} \quad \text { with } \theta \in\left[-\frac{\pi}{2} ; \frac{\pi}{2}\right]
$$

and

$$
\frac{\gamma}{2}-i v=\sqrt{\frac{\gamma^{2}}{4}+v^{2}} e^{-i \theta} \quad \text { with }-\theta \in\left[-\frac{\pi}{2} ; \frac{\pi}{2}\right]
$$

Then we have

$$
e^{-i \pi / 4}\left(\frac{\gamma}{2}-i v\right) \sqrt{\frac{\gamma}{2}+i v}=\sqrt{\frac{\gamma^{2}}{4}+v^{2} e^{-i\left(\frac{\theta}{2}+\frac{\pi}{4}\right)}} \quad \text { with }-\frac{\theta}{2}-\frac{\pi}{4} \in\left[-\frac{\pi}{2} ; 0\right] .
$$

From the fact that $\operatorname{Re}\left(e^{-i \pi / 4}\left(\frac{\gamma}{2}-i v\right) \sqrt{\left.\frac{\gamma}{2}+i v\right)} \geq 0\right.$ and by using the following equality

$$
e^{-i \pi / 4} \int_{0}^{+\infty} e^{-\gamma t} \overline{\psi_{t}} \partial_{t}^{1 / 2} \psi d t=\left.\frac{e^{-i \pi / 4}}{2 \pi} \int_{-\infty}^{+\infty}\left(\frac{\gamma}{2}-i v\right) \sqrt{\frac{\gamma}{2}+i v \mid} \mathcal{L}(\psi)\left(i v+\frac{\gamma}{2}\right)\right|^{2} d v
$$

we obtain inequality (ii). 
Remark 1 Let $\phi \in \mathbf{H}^{\mathbf{1} / \mathbf{4}}\left(\mathbf{t}_{\mathbf{1}}, \mathbf{t}_{2}\right)$ be a function extended by zero outside the interval $\left[t_{1}, t_{2}\right]$. The following hold:

- $\operatorname{Re}\left(e^{ \pm i \pi / 4} \int_{t_{1}}^{t_{2}} e^{-\gamma t} \bar{\phi} \partial_{t}^{1 / 2} \phi d t\right) \geq 0$.

- By continuity of the term $\bar{\phi} \partial_{t}^{1 / 2} \phi$ and by dividing the term $\operatorname{Re}\left(e^{ \pm i \pi / 4} \int_{t_{1}}^{t_{2}} e^{-\gamma t} \bar{\phi} \partial_{t}^{1 / 2} \phi d t\right)$ by $t_{2}-t_{1}$, we have when $t_{2} \rightarrow t_{1}, \operatorname{Re}\left(e^{ \pm i \pi / 4} e^{-\gamma t} \overline{\phi(t)} \partial_{t}^{1 / 2} \phi(t) d t\right) \geq 0$ for all $t \geq 0$.

Finally, we introduce our last technical result.

Lemma 2 Let $w \in \mathbf{H}^{\mathbf{1}}(\boldsymbol{\Omega})$ be a function defined from $\left.\boldsymbol{\Omega}=\right]-\infty, \mathbf{0}[$ to $\mathbb{C}$. Then we have the following inequality:

$$
|w(0)|^{2} \leq 2\|w\|_{L^{2}(\Omega)}\left\|w_{x}\right\|_{L^{2}(\Omega)} .
$$

Proof Let $y \in \boldsymbol{\Omega}$, we have

$$
\int_{y}^{0} \frac{d}{d x}[w(x)]^{2} d x=w^{2}(0)-w^{2}(y)
$$

which gives

$$
w^{2}(0)=w^{2}(y)+2 \int_{y}^{0} w_{x}(x) w(x) d x
$$

Since $w \in \mathbf{H}^{\mathbf{1}}(\boldsymbol{\Omega})$, we have $\lim _{y \rightarrow-\infty} w(y)=0$. Then we have when $y \rightarrow-\infty$

$$
|w(0)|^{2} \leq 2 \int_{-\infty}^{0}\left|w_{x}(x)\right||w(x)| d x
$$

By using the Cauchy-Schwarz inequality, we get

$$
|w(0)|^{2} \leq 2\|w\|_{L^{2}(\Omega)}\left\|w_{x}\right\|_{L^{2}(\Omega)} .
$$

\section{Semigroup associated with NLS equation}

In the following result, we show that the Schrödinger equation can be characterized by an autonomous dynamical system.

Proposition 1 NLS equation (2)-(4)-(6) is equivalent to an autonomous dynamical system with a semigroup $(S(t))_{t \geq 0}$ on $\mathbf{H}^{\mathbf{1}}(\boldsymbol{\Omega})$.

Proof Let us show that for any $u$ solution to NLS equation (2)-(4) associated with the initial data $u_{0}$, the following function

$$
\begin{gathered}
S(t): \mathbf{H}^{\mathbf{1}}(\boldsymbol{\Omega}) \rightarrow \mathbf{H}^{\mathbf{1}}(\boldsymbol{\Omega}) \\
u_{0} \mapsto u(t),
\end{gathered}
$$

defines a semigroup.

To prove $S(t+s)=S(t) S(s)$, we define the solutions $u(t)$ associated with the initial data $u_{0}$ and $v(t)$ associated with the initial data $u(s)$. Then it suffices to show that $u$ and $v$ satisfy 
the boundary condition (4), and hence we will conclude that $v(t)=u(t+s)$. For this aim, we check that $u(t+s)$ is a solution of (2) at time $t+s$. Indeed, using the expression of the phase function $\mathbb{V}$ in (4), we see that by using the change of variable we can obtain

$$
\begin{aligned}
\partial_{n} u(0, t+s)= & -\sqrt{\frac{2}{\pi}} e^{-i \pi / 4} e^{-\gamma(t+s)} e^{i \mathbb{V}(0, t+s)} \partial_{t} \int_{-\infty}^{t+s} \frac{e^{\gamma \tau} e^{-i \mathbb{V}(0, \tau)} u(0, \tau)}{\sqrt{t+s-\tau}} d \tau \\
= & -\sqrt{\frac{2}{\pi}} e^{-i \pi / 4} e^{-\gamma(t+s)} e^{i \mathbb{V}(0, t+s)} \\
& \times \partial_{t} \int_{-\infty}^{t} \frac{e^{\gamma(\tau+s)} e^{-i \mathbb{V}(0, \tau+s)} u(0, \tau+s)}{\sqrt{t-\tau}} d \tau \\
= & -\sqrt{\frac{2}{\pi}} e^{-i \pi / 4} e^{-\gamma t} \exp \left(i \int_{0}^{t+s} g\left(|u(0, \tau)|^{2}\right) d \tau\right) \\
& \times \partial_{t} \int_{-\infty}^{t} \frac{e^{\gamma \tau} \exp \left(-i \int_{0}^{\tau+s} g\left(|u(0, \theta)|^{2}\right) d \theta\right) u(0, \tau+s)}{\sqrt{t-\tau}} d \tau \\
= & -\sqrt{\frac{2}{\pi}} e^{-i \pi / 4} e^{-\gamma t} \exp \left(i \int_{-s}^{t} g\left(|u(0, \tau+s)|^{2}\right) d \tau\right) \\
& \times \partial_{t} \int_{-\infty}^{t} \frac{e^{\gamma \tau} \exp \left(-i \int_{-s}^{\tau} g\left(|u(0, \theta+s)|^{2}\right) d \theta\right) u(0, \tau+s)}{\sqrt{t-\tau}} d \tau \\
= & -\sqrt{\frac{2}{\pi}} e^{-i \pi / 4} e^{-\gamma t} \exp \left(i \int_{0}^{t} g\left(|u(0, \tau+s)|^{2}\right) d \tau\right) \\
& \times \partial_{t} \int_{-\infty}^{t} \frac{e^{\gamma \tau} \exp \left(-i \int_{0}^{\tau} g\left(|u(0, \theta+s)|^{2}\right) d \theta\right) u(0, \tau+s)}{\sqrt{t-\tau}} d \tau .
\end{aligned}
$$

So that we have

$$
\begin{aligned}
\partial_{n} v(0, t)= & -\sqrt{\frac{2}{\pi}} e^{-i \pi / 4} e^{-\gamma t} \exp \left(i \int_{0}^{t} g\left(|v(0, \tau)|^{2}\right) d \tau\right) \\
& \times \partial_{t} \int_{-\infty}^{t} \frac{e^{\gamma \tau} \exp \left(-i \int_{0}^{\tau} g\left(|v(0, \theta)|^{2}\right) d \theta\right) \nu(0, \tau)}{\sqrt{t-\tau}} d \tau .
\end{aligned}
$$

By taking into account the uniqueness of the solution, we conclude that $v(t)=u(t+s)$ and the proof is complete.

For the sake of simplicity, we make a change of the unknown solution to NLS equation (2)-(4)-(6) as follows:

$$
v(x, t)=\exp (-i \mathbb{V}(0, t)) u(x, t) .
$$

Then NLS equation (2)-(4)-(6) can be rewritten in the following form:

$$
\left\{\begin{array}{l}
i v_{t}+\frac{1}{2} v_{x x}+q \delta_{a} v+\left(g\left(|v(x, t)|^{2}\right)-g\left(|v(0, t)|^{2}\right)\right) v+i \gamma v \\
\quad=\exp (-i \mathbb{V}(0, t)) f \quad \text { in } \Omega \times \mathbb{R}_{+}, \\
\partial_{n} v(0, t)+\sqrt{2} e^{-i \pi / 4} e^{-\gamma t} \partial_{t}^{1 / 2}\left(e^{\gamma t} v(0, t)\right)=0, \quad t \in \mathbb{R}_{+}, \\
v(x, 0)=u_{0}(x), \quad x \in \Omega .
\end{array}\right.
$$


Remark 2 From the previous change of variable (8), we can easily check the following properties:

(i) $|v(x, t)|=|u(x, t)|$,

(ii) $u(x, t)=\exp \left(i \int_{0}^{t} g\left(|v(0, s)|^{2}\right) d s\right) v(x, t)$,

(iii) $\|v(t)\|_{H^{s}(\Omega)}=\|u(t)\|_{H^{s}(\Omega)}$ for all $s \geq 0$.

Proposition 2 The semigroup $(S(t))_{t \geq 0}$ defined by (7) is continuous on the bounded subsets of $\mathbf{H}^{\mathbf{1}}(\boldsymbol{\Omega})$ for the strong topology of $\mathbf{L}^{2}(\boldsymbol{\Omega})$.

Proof Let $v(t)$ and $\tilde{v}(t)$ be two solutions verifying NLS equation (9). We set $w(t)=v(t)-\tilde{v}(t)$ with the initial data $w(0)=u_{0}-\tilde{u}_{0}$. Then we get

$$
\begin{aligned}
i w_{t}+ & \frac{1}{2} w_{x x}+q \delta_{a} w+g\left(|v|^{2}\right) v-g\left(|\tilde{v}|^{2}\right) \tilde{v} \\
& -g\left(|v(0, t)|^{2}\right) v+g\left(|\tilde{v}(0, t)|^{2}\right) \tilde{v}+i \gamma w \\
= & (\exp (-i \mathbb{V}(0, t))-\exp (-i \tilde{\mathbb{V}}(0, t))) f,
\end{aligned}
$$

where $\mathbb{V}(0, t)=\int_{0}^{t} g\left(|v(0, s)|^{2}\right) d s$ and $\tilde{\mathbb{V}}(0, t)=\int_{0}^{t} g\left(|\tilde{v}(0, s)|^{2}\right) d s$. The boundary condition is given by

$$
\partial_{n} w(0, t)+\sqrt{2} e^{-i \pi / 4} e^{-\gamma t} \partial_{t}^{1 / 2}\left(e^{\gamma t} w(0, t)\right)=0, \quad t \in \mathbb{R}_{+} .
$$

Now, multiplying equation (10) by $\bar{w}$ and taking the imaginary part, we get

$$
\begin{aligned}
\frac{1}{2} \frac{d}{d t}\|w\|_{L^{2}(\Omega)}^{2}+\gamma\|w\|_{L^{2}(\Omega)}^{2} \\
=-\operatorname{Im} m\left(\partial_{n} w(0, t) \bar{w}(0, t)\right)-\mathcal{I} m\left(\int_{\Omega}\left(g\left(|v|^{2}\right) v-g\left(|\tilde{v}|^{2}\right) \tilde{v}\right) \bar{w} d x\right) \\
\quad+\mathcal{I} m\left(\int_{\Omega}\left(g\left(|v(0, t)|^{2}\right) v-g\left(|\tilde{v}(0, t)|^{2}\right) \tilde{v}\right) \bar{w} d x\right) \\
\quad+\operatorname{I} m\left((\exp (-i \mathbb{V}(0, t))-\exp (-i \tilde{\mathbb{V}}(0, t))) \int_{\Omega} f \bar{w} d x\right)
\end{aligned}
$$

Observe that

$$
-\mathcal{I} m\left(\partial_{n} w(0, t) \bar{w}(0, t)\right)=-\sqrt{2} \mathcal{R} e\left(e^{i \pi / 4} \overline{e^{\gamma t} w(0, t)} \partial_{t}^{1 / 2}\left(e^{\gamma t} w(0, t)\right)\right)
$$

Based on this identity and by using Remark 1 , we can see that the term $-\mathcal{I} m\left(\partial_{n} w(0, t) \bar{w}(0\right.$, $t)) \leq 0$. This fact is used to bound the first right term of (12).

Next, other right terms of (12) are shown to be bounded by using the Cauchy-Schwarz inequality, the continuity of injection of $\mathbf{H}^{\mathbf{1}}(\boldsymbol{\Omega})$ in $\mathbf{L}^{\infty}(\boldsymbol{\Omega})$ and by taking into account assumptions (3) on the function $g$. According to these considerations, we derive

$$
-\mathcal{I} m\left(\int_{\Omega}\left(g\left(|v|^{2}\right) v-g\left(|\tilde{v}|^{2}\right) \tilde{v}\right) \bar{w} d x\right) \leq K\|w(t)\|_{L^{2}(\Omega)}^{2}
$$


and the following majorization by using Lemma 2

$$
\begin{aligned}
& \mathcal{I} m\left(\int_{\Omega}\left(g\left(|v(0, t)|^{2}\right) v-g\left(|\tilde{v}(0, t)|^{2}\right) \tilde{v}\right) \bar{w} d x\right) \\
& \quad+\mathcal{I} m\left((\exp (-i \mathbb{V}(0, t))-\exp (-i \tilde{\mathbb{V}}(0, t))) \int_{\Omega} f \bar{w} d x\right) \leq K\|w(t)\|_{L^{2}(\Omega)}^{2}
\end{aligned}
$$

From the above inequalities it follows

$$
\frac{d}{d t}\|w(t)\|_{L^{2}(\Omega)}^{2}+\gamma\|w(t)\|_{L^{2}(\Omega)}^{2} \leq K\|w(t)\|_{L^{2}(\Omega)}^{2} .
$$

By using Gronwall's lemma and Lemma 1, we get

$$
\|w(t)\|_{L^{2}(\Omega)}^{2} \leq e^{(K-\gamma) t}\|w(0)\|^{2} .
$$

Using Remark 2, we have

$$
\begin{aligned}
u(t)-\tilde{u}(t)= & \exp \left(i \int_{0}^{t} g\left(|v(0, s)|^{2}\right) d s\right) v(x, t) \\
& -\exp \left(i \int_{0}^{t} g\left(|\tilde{v}(0, s)|^{2}\right) d s\right) \tilde{v}(x, t) .
\end{aligned}
$$

Then it follows

$$
\begin{aligned}
& \|u(t)-\tilde{u}(t)\|_{L^{2}(\Omega)} \\
& \leq\|v(t)-\tilde{v}(t)\|_{L^{2}(\Omega)} \\
& \quad+\|\tilde{v}(t)\|_{L^{2}(\Omega)}\left|\exp \left(i \int_{0}^{t} g\left(|v(0, s)|^{2}\right) d s\right)-\exp \left(i \int_{0}^{t} g\left(|\tilde{v}(0, s)|^{2}\right) d s\right)\right| \\
& \leq\|v(t)-\tilde{v}(t)\|_{L^{2}(\Omega)}+\|\tilde{v}(t)\|_{L^{2}(\Omega)} \int_{0}^{t}\left|g\left(|v(0, s)|^{2}\right)-g\left(|\tilde{v}(0, s)|^{2}\right)\right| d s .
\end{aligned}
$$

By using the mean value theorem and assumption (3) on the function $g$, we obtain

$$
\begin{aligned}
& \|u(t)-\tilde{u}(t)\|_{L^{2}(\Omega)} \\
& \quad \leq\|w(t)\|_{L^{2}(\Omega)}+K\|\tilde{v}(t)\|_{L^{2}(\Omega)} \sup _{t \in[0, T]}[|w(0, t)|(|v(0, t)|+|\tilde{v}(0, t)|)] .
\end{aligned}
$$

By using Lemma 2, we get

$$
\begin{aligned}
\|u(t)-\tilde{u}(t)\|_{L^{2}(\Omega)} & \leq \sup _{t \in[0, T]}\left[\|w(t)\|_{L^{2}(\Omega)}+K\|w(t)\|_{L^{2}(\Omega)}^{\frac{1}{2}}\right] \\
& \leq K \sup _{t \in[0, T]}\left[\|w(t)\|_{L^{2}(\Omega)}^{\frac{1}{2}}\right] .
\end{aligned}
$$

Finally, by taking the supremum on the left-hand side of the above inequality and by using inequality (14), we obtain

$$
\sup _{t \in[0, T]}\left[\|u(t)-\tilde{u}(t)\|_{L^{2}(\Omega)}\right] \leq K \sup _{t \in[0, T]}\left[e^{\frac{K-\gamma}{4} t}\right]\left\|u_{0}-\tilde{u}_{0}\right\|_{L^{2}(\Omega)}^{\frac{1}{2}},
$$


which can establish the continuity of the semigroup $(S(t))_{t \geq 0}$ on bounded subsets of $\mathbf{H}^{\mathbf{1}}(\boldsymbol{\Omega})$ for the strong topology of $\mathbf{L}^{2}(\Omega)$.

\section{Bounded absorbing set in $\mathrm{H}^{1}(\Omega)$}

In the sequel, we show the dissipativity of the autonomous dynamical system which was previously defined by the semigroup $\left(\mathbf{H}^{\mathbf{1}}(\boldsymbol{\Omega}),(\mathbf{S}(\mathbf{t}))_{\mathbf{t} \geq \mathbf{0}}\right)$.

Theorem 2 There exists a bounded subset denoted by $\mathcal{B}_{a b}$ of $\mathbf{H}^{\mathbf{1}}(\boldsymbol{\Omega})$ such that for any bounded subset $B$ of $\mathbf{H}^{\mathbf{1}}(\boldsymbol{\Omega})$, there exists a time $t^{\star}(B)>0$ satisfying

$$
S(t) B \subset \mathcal{B}_{a b} \text { for all } t \geq t^{\star}(B)
$$

Moreover, this bounded subset $\mathcal{B}_{a b}$ can be determined as a closed positive-invariant set.

Proof First, we prove this result in $\mathbf{L}^{2}(\boldsymbol{\Omega})$.

Bounded absorbing set in $\mathbf{L}^{2}(\boldsymbol{\Omega})$ :

Multiply equation (9) by $-i \bar{v}$, integrate in the space domain $\Omega$, and then integrate by parts the second term and consider the real part of the resulting equation

$$
\begin{aligned}
& \frac{1}{2} \frac{d}{d t}\|v(t)\|_{L^{2}(\Omega)}^{2}+\gamma\|v(t)\|_{L^{2}(\Omega)}^{2} \\
& \quad=\frac{1}{2} \mathcal{R} e\left(\overline{i v(0, t)} \partial_{h} v(0, t)\right)+\mathcal{I} m\left(\exp (-i \mathbb{V}(0, t)) \int_{-\infty}^{0} f \bar{v} d x\right) .
\end{aligned}
$$

Using the Cauchy-Schwarz and Young's inequalities, we get

$$
\frac{d}{d t}\|v(t)\|_{L^{2}(\Omega)}^{2}+\gamma\|v(t)\|_{L^{2}(\Omega)}^{2} \leq \frac{1}{\gamma}\|f\|_{L^{2}(\Omega)}^{2}+\frac{1}{2} \mathcal{R} e\left(\overline{i v(0, t)} \partial_{n} v(0, t)\right) .
$$

Using Gronwall's lemma, we obtain

$$
\begin{aligned}
\|v(t)\|_{L^{2}(\Omega)}^{2} \leq & e^{-\gamma t}\left\|u_{0}\right\|_{L^{2}(\Omega)}^{2}+\frac{1-e^{-\gamma t}}{\gamma^{2}}\|f\|_{L^{2}(\Omega)}^{2} \\
& +\frac{e^{-\gamma t}}{2} \int_{0}^{t} e^{\gamma s} \mathcal{R} e\left(i \overline{\nu(0, s)} \partial_{n} v(0, s)\right) d s .
\end{aligned}
$$

Using the boundary condition for NLS equation (9) and Lemma 1, we can show that $\frac{e^{-\gamma t}}{2} \int_{0}^{t} e^{\gamma s} \mathcal{R} e\left(i \overline{v(0, s)} \partial_{n} v(0, s)\right) d s \leq 0$. Hence, we obtain

$$
\|v(t)\|_{L^{2}(\Omega)}^{2} \leq e^{-\gamma t}\left\|u_{0}\right\|_{L^{2}(\Omega)}^{2}+\frac{1-e^{-\gamma t}}{\gamma^{2}}\|f\|_{L^{2}(\Omega)}^{2} .
$$

Then there exists $t_{0}=\frac{2}{\gamma} \ln \left(\frac{\gamma\left\|u_{0}\right\|_{L^{2}(\Omega)}}{\|f\|_{L^{2}(\Omega)}}\right)>0$ such that for $t \geq t_{0}$, we have

$$
\|u(t)\|_{L^{2}(\Omega)}^{2}=\|v(t)\|_{L^{2}(\Omega)}^{2} \leq \frac{2}{\gamma^{2}}\|f\|_{L^{2}(\Omega)}^{2}=M_{0}^{2} .
$$


We consider in the following $t \geq t_{0}$. We multiply the first equality of (9) by $\overline{v_{t}+\gamma \nu}$, and we integrate in the space domain $\Omega$. By considering the real part, we get

$$
\begin{aligned}
\frac{d}{d t} \Psi(v(t))+2 \gamma \Psi(v(t)) \\
=2 \operatorname{Re} e\left(\overline{v_{t}(0, t)+\gamma v(0, t)} \partial_{n} v(0, t)\right) \\
\quad-2 \gamma g\left(|v(0, t)|^{2}\right)\|v\|_{L^{2}(\Omega)}^{2}+4 \gamma \int_{-\infty}^{0}\left(|v|^{2} g\left(|v|^{2}\right)-G\left(|v|^{2}\right)\right) d x \\
\quad-g\left(|v(0, t)|^{2}\right) \frac{d}{d t}\|v\|_{L^{2}(\Omega)}^{2}+4 \gamma \mathcal{R} e\left(\exp (-i \mathbb{V}(0, t)) \int_{-\infty}^{0} f \bar{v} d x\right) \\
\quad-4 \mathcal{R} e\left(i|v(0, t)|^{2} \exp (-i \mathbb{V}(0, t)) \int_{-\infty}^{0} f \bar{v} d x\right),
\end{aligned}
$$

where

$$
\begin{aligned}
\Psi(v(t))= & \left\|v_{x}\right\|_{L^{2}(\Omega)}^{2}-2 q|v(a, t)|^{2}-2 \int_{\Omega} G\left(|v|^{2}\right) d x \\
& +4 \mathcal{R} e\left(\exp (-i \mathbb{V}(0, t)) \int_{-\infty}^{0} f \bar{v} d x\right),
\end{aligned}
$$

where $G$ is defined by (3).

We first need to majorize $\left\|v_{x}\right\|_{L^{2}(\Omega)}^{2}$ in terms of $\Psi(v(t))$. Indeed, we have by (20)

$$
\begin{aligned}
\left\|v_{x}\right\|_{L^{2}(\Omega)}^{2}= & \Psi(v(t))+2 q|v(a, t)|^{2}+2 \int_{\Omega} G\left(|v|^{2}\right) d x \\
& -4 \mathcal{R} e\left(\exp (-i \mathbb{V}(0, t)) \int_{-\infty}^{0} f \bar{v} d x\right) .
\end{aligned}
$$

From here, we stress out that $M$ can be any positive constant depending only on $q, \gamma, \alpha_{1}$, $\alpha_{2}$ and $\|f\|_{L^{2}(\Omega)}$. But this quantity is independent of $u_{0}$.

Now, by Agmon's, Young's inequalities and inequality (18), we have

$$
2 q|v(a, t)|^{2} \leq 2|q|\|v\|_{L^{\infty}(\Omega)}^{2} \leq 2|q| M_{0}\left\|v_{x}\right\|_{L^{2}(\Omega)} \leq \frac{1}{4}\left\|v_{x}\right\|_{L^{2}(\Omega)}^{2}+M .
$$

By considering (3) and by using the Gagliardo-Nirenberg and Young's inequalities, we obtain

$$
\int_{\Omega} G\left(|v|^{2}\right) d x \leq \alpha_{2}\left(\|v\|_{L^{2}(\Omega)}^{2}+\int_{\Omega}|v|^{2+2 \theta}\right) \leq \frac{1}{8}\left\|v_{x}\right\|_{L^{2}(\Omega)}^{2}+M .
$$

The last term of (21) can be bounded by using the Cauchy-Schwarz inequality as follows:

$$
-4 \mathcal{R} e\left(\exp (-i \mathbb{V}(0, t)) \int_{-\infty}^{0} f \bar{v} d x\right) \leq 4\|f\|_{L^{2}(\Omega)} M_{0} \leq M
$$

Hence, by using the above inequalities (22)-(24), we get

$$
\left\|v_{x}\right\|_{L^{2}(\Omega)}^{2} \leq 2 \Psi(v(t))+M
$$


Next, we majorize the second member of equality (19).

Majorization of the term $4 \gamma \int_{-\infty}^{0}\left(|v|^{2} g\left(|v|^{2}\right)-G\left(|v|^{2}\right)\right) d x$ :

We use the inequalities of Gagliardo-Nirenberg and Young combined with (3) and (25) to obtain

$$
\begin{aligned}
& 4 \gamma \int_{-\infty}^{0}\left(|v|^{2} g\left(|v|^{2}\right)-G\left(|v|^{2}\right)\right) d x \\
& \quad \leq 4 \gamma\left(\alpha_{1}+\alpha_{2}\right)\left(\|v\|_{L^{2}(\Omega)}^{2}+\int_{\Omega}|v|^{2+2 \theta} d x\right) \\
& \quad \leq 4 \gamma\left(\alpha_{1}+\alpha_{2}\right)\left(M_{0}^{2}+C M_{0}^{2+\theta}\left\|v_{x}\right\|_{L^{2}(\Omega)}^{\theta}\right) \\
& \quad \leq M\left(1+\left\|v_{x}\right\|_{L^{2}(\Omega)}^{\theta}\right) \\
& \quad \leq \frac{\gamma}{16}\left\|v_{x}\right\|_{L^{2}(\Omega)}^{2}+M \leq \frac{\gamma}{8} \Psi(v(t))+M .
\end{aligned}
$$

Majorization of the term $-2 \gamma g\left(|v(0, t)|^{2}\right)\|v\|_{L^{2}(\Omega)}^{2}$ :

We use Young's inequality and Lemma 2 combined with (3) and (25) to get

$$
\begin{aligned}
-2 \gamma g\left(|v(0, t)|^{2}\right)\|v\|_{L^{2}(\Omega)}^{2} & \leq M\left(1+\left\|v_{x}\right\|_{L^{2}(\Omega)}^{\theta}\right) \\
& \leq \frac{\gamma}{16}\left\|v_{x}\right\|_{L^{2}(\Omega)}^{2}+M \leq \frac{\gamma}{8} \Psi(v(t))+M .
\end{aligned}
$$

Majorization of the term $-4 \mathcal{R} e\left(i|v(0, t)|^{2} \exp (-i \mathbb{V}(0, t)) \int_{-\infty}^{0} f \bar{v} d x\right)$ :

We use Young's inequality and Lemma 2 combined with (18) and (25) to obtain

$$
\begin{array}{rl}
-4 & \mathcal{R} e\left(i|v(0, t)|^{2} \exp (-i \mathbb{V}(0, t)) \int_{-\infty}^{0} f \bar{v} d x\right) \\
& \leq M|v(0, t)|^{2} \leq 2 M\|v\|_{L^{2}(\Omega)}\left\|v_{x}\right\|_{L^{2}(\Omega)} \leq M\left\|v_{x}\right\|_{L^{2}(\Omega)} \\
& \leq \frac{\gamma}{8}\left\|v_{x}\right\|_{L^{2}(\Omega)}^{2}+M \leq \frac{\gamma}{4} \Psi(v(t))+M .
\end{array}
$$

Majorization of the term $-g\left(|v(0, t)|^{2}\right) \frac{d}{d t}\|v\|_{L^{2}(\Omega)}^{2}$ :

For this majorization, we use the following fact.

Fact 1 By using the boundary condition of NLS equation (9), it follows from (15) and Remark 1 that

$$
\mathcal{I} m\left(\exp (-i \mathbb{V}(0, t)) \int_{-\infty}^{0} f \bar{v} d x\right)-\frac{1}{2} \frac{d}{d t}\|v\|_{L^{2}(\Omega)}^{2} \geq 0 .
$$

Now, we write the term $-g\left(|v(0, t)|^{2}\right) \frac{d}{d t}\|v\|_{L^{2}(\Omega)}^{2}$ as follows:

$$
\begin{aligned}
-g & \left(|v(0, t)|^{2}\right) \frac{d}{d t}\|v\|_{L^{2}(\Omega)}^{2} \\
= & 2 g\left(|v(0, t)|^{2}\right)\left(\mathcal{I} m\left(\exp (-i \mathbb{V}(0, t)) \int_{-\infty}^{0} f \bar{v} d x\right)-\frac{1}{2} \frac{d}{d t}\|v\|_{L^{2}(\Omega)}^{2}\right) \\
& -2 g\left(|v(0, t)|^{2}\right) \mathcal{I} m\left(\exp (-i \mathbb{V}(0, t)) \int_{-\infty}^{0} f \bar{v} d x\right) .
\end{aligned}
$$


Taking into account the previous Fact 1, assumption (3) on the function $g$, Lemma 2, then by using Young's inequality and (25), we obtain

$$
\begin{aligned}
-g\left(|v(0, t)|^{2}\right) \frac{d}{d t}\|v\|_{L^{2}(\Omega)}^{2} \\
\leq 2 \alpha_{2}\left(1+|v(0, t)|^{2 \theta}\right)\left(\mathcal{I} m\left(\exp (-i \mathbb{V}(0, t)) \int_{-\infty}^{0} f \bar{v} d x\right)-\frac{1}{2} \frac{d}{d t}\|v\|_{L^{2}(\Omega)}^{2}\right) \\
\quad+\alpha_{2} M\left(1+|v(0, t)|^{2 \theta}\right) \\
\leq 2 \alpha_{2}\left(1+2 M_{0}^{\theta}\left\|v_{x}\right\|_{L^{2}(\Omega)}^{\theta}\right)\left(\mathcal{I} m\left(\exp (-i \mathbb{V}(0, t)) \int_{-\infty}^{0} f \bar{v} d x\right)-\frac{1}{2} \frac{d}{d t}\|v\|_{L^{2}(\Omega)}^{2}\right) \\
\quad+\alpha_{2} M\left(1+2 M_{0}^{\theta}\left\|v_{x}\right\|_{L^{2}(\Omega)}^{\theta}\right) \\
\leq\left(\epsilon\left\|v_{x}\right\|_{L^{2}(\Omega)}^{2}+M\right)\left(\mathcal{I} m\left(\exp (-i \mathbb{V}(0, t)) \int_{-\infty}^{0} f \bar{v} d x\right)-\frac{1}{2} \frac{d}{d t}\|v\|_{L^{2}(\Omega)}^{2}\right) \\
\quad+\frac{\gamma}{2} \Psi(v(t))+M .
\end{aligned}
$$

Using (19), (24), (26), (27), (28) and (30), we obtain

$$
\begin{aligned}
& \frac{d}{d t} \Psi(v(t))+\gamma \Psi(v(t)) \\
& \leq M+2 \mathcal{R} e\left(\overline{v_{t}(0, t)+\gamma \nu(0, t)} \partial_{n} v(0, t)\right) \\
& \quad+\left(\epsilon\left\|v_{x}\right\|_{L^{2}(\Omega)}^{2}+M\right)\left(\mathcal{I} m\left(\exp (-i \mathbb{V}(0, t)) \int_{-\infty}^{0} f \bar{v} d x\right)-\frac{1}{2} \frac{d}{d t}\|v\|_{L^{2}(\Omega)}^{2}\right) .
\end{aligned}
$$

We multiply this inequality by $e^{\gamma t}$ to have

$$
\begin{aligned}
& \frac{d}{d t}\left(e^{\gamma t} \Psi(v(t))\right) \\
& \leq M e^{\gamma t}+2 \mathcal{R} e\left(\overline{\left(e^{\gamma t} v(0, t)\right)_{t}} \partial_{n} v(0, t)\right) \\
& +\left(e^{\frac{\gamma t}{2}} \epsilon\left\|v_{x}\right\|_{L^{2}(\Omega)}^{2}+M e^{\frac{\gamma t}{2}}\right) e^{\frac{\gamma t}{2}} \\
& \times\left(\mathcal{I} m\left(\exp (-i \mathbb{V}(0, t)) \int_{-\infty}^{0} f \bar{v} d x\right)-\frac{1}{2} \frac{d}{d t}\|v\|_{L^{2}(\Omega)}^{2}\right) \\
& \leq M e^{\gamma t}+2 \mathcal{R} e\left(\overline{\left(e^{\gamma t} v(0, t)\right)_{t}} \partial_{n} v(0, t)\right) \\
& +\left(\epsilon e^{\frac{\gamma t}{2}} \sup _{t \in\left[t_{0}, T\right]}\left[e^{\frac{\gamma t}{2}}\left\|v_{x}\right\|_{L^{2}(\Omega)}^{2}\right]+M e^{\gamma t}\right) \\
& \times\left(\mathcal{I} m\left(\exp (-i \mathbb{V}(0, t)) \int_{-\infty}^{0} f \bar{v} d x\right)-\frac{1}{2} \frac{d}{d t}\|v\|_{L^{2}(\Omega)}^{2}\right) .
\end{aligned}
$$

From the above inequality it can be easily seen that

$$
\begin{aligned}
& \frac{d}{d t}\left(e^{\gamma t} \Psi(v(t))+\frac{1}{2}\left(\epsilon e^{\frac{\gamma t}{2}} \sup _{t \in\left[t_{0}, T\right]}\left[e^{\frac{\gamma t}{2}}\left\|v_{x}\right\|_{L^{2}(\Omega)}^{2}\right]+M e^{\gamma t}\right)\|v\|_{L^{2}(\Omega)}^{2}\right) \\
& \quad \leq\left(M+\frac{\gamma}{2} M\|v\|_{L^{2}(\Omega)}^{2}\right) e^{\gamma t}+2 \mathcal{R} e\left(\overline{\left(e^{\gamma t} v(0, t)\right)_{t}} \partial_{n} v(0, t)\right)
\end{aligned}
$$




$$
\begin{aligned}
& +\left(\epsilon e^{\frac{\gamma t}{2}} \sup _{t \in\left[t_{0}, T\right]}\left[e^{\frac{\gamma t}{2}}\left\|v_{x}\right\|_{L^{2}(\Omega)}^{2}\right]+M e^{\gamma t}\right) \mathcal{I} m\left(\exp (-i \mathbb{V}(0, t)) \int_{-\infty}^{0} f \bar{v} d x\right) \\
& +\frac{1}{4} \epsilon \gamma\|v\|_{L^{2}(\Omega)}^{2} e^{\frac{\gamma t}{2}} \sup _{t \in\left[t_{0}, T\right]}\left[e^{\frac{\gamma t}{2}}\left\|v_{x}\right\|_{L^{2}(\Omega)}^{2}\right] .
\end{aligned}
$$

Then we use the above inequality combined with (18) and (24) to get

$$
\begin{aligned}
& \frac{d}{d t}\left(e^{\gamma t} \Psi(v(t))+\frac{1}{2}\left(\epsilon e^{\frac{\gamma t}{2}} \sup _{t \in\left[t_{0}, T\right]}\left[e^{\frac{\gamma t}{2}}\left\|v_{x}\right\|_{L^{2}(\Omega)}^{2}\right]+M e^{\gamma t}\right)\|v\|_{L^{2}(\Omega)}^{2}\right) \\
& \quad \leq 2 \mathcal{R} e\left(\overline{\left(e^{\gamma t} v(0, t)\right)_{t}} \partial_{h} v(0, t)\right)+\epsilon M e^{\frac{\gamma t}{2}} \sup _{t \in\left[t_{0}, T\right]}\left[e^{\frac{\gamma t}{2}}\left\|v_{x}\right\|_{L^{2}(\Omega)}^{2}\right]+M e^{\gamma t}
\end{aligned}
$$

We integrate the above inequality between $t_{0}$ and $t$ with $t \in\left[t_{0}, T\right]$ to have

$$
\begin{aligned}
e^{\gamma t} \Psi(v(t)) \leq & e^{\gamma t_{0}} \Psi\left(v\left(t_{0}\right)\right)+M\left(e^{\gamma t}-e^{\gamma t_{0}}\right) \\
& +2 \int_{t_{0}}^{t} \mathcal{R} e\left(\overline{\left(e^{\gamma s} v(0, s)\right)_{s}} \partial_{n} \nu(0, s)\right) d s \\
& +\frac{1}{2}\left(\epsilon e^{\frac{\gamma t_{0}}{2}} \sup _{t \in\left[t_{0}, T\right]}\left[e^{\frac{\gamma t}{2}}\left\|v_{x}\right\|_{L^{2}(\Omega)}^{2}\right]+M e^{\gamma t_{0}}\right)\left\|v\left(t_{0}\right)\right\|_{L^{2}(\Omega)}^{2} \\
& +\epsilon M\left(e^{\frac{\gamma t}{2}}-e^{\frac{\gamma t_{0}}{2}}\right) \sup _{t \in\left[t_{0}, T\right]}\left[e^{\frac{\gamma t}{2}}\left\|v_{x}\right\|_{L^{2}(\Omega)}^{2}\right] .
\end{aligned}
$$

By using the boundary condition of NLS equation (9) and Lemma 1, we have

$$
\begin{aligned}
& 2 \int_{t_{0}}^{t} \mathcal{R} e\left(\overline{\left(e^{\gamma s} v(0, s)\right)_{s}} \partial_{h} v(0, s)\right) d s \\
& \quad=-2 \sqrt{2} \mathcal{R} e\left(e^{-i \pi / 4} \int_{t_{0}}^{t} e^{-\gamma s} \overline{\partial_{s}\left(e^{\gamma s} v(0, s)\right)} \partial_{s}^{1 / 2}\left(e^{\gamma s} v(0, s)\right) d s\right) \leq 0
\end{aligned}
$$

which gives

$$
\begin{aligned}
e^{\gamma t} \Psi(v(t)) \leq & e^{\gamma t_{0}} \Psi\left(v\left(t_{0}\right)\right)+\frac{1}{2}\left(\epsilon e^{\frac{\gamma t_{0}}{2}} \sup _{t \in\left[t_{0}, T\right]}\left[e^{\frac{\gamma t}{2}}\left\|v_{x}\right\|_{L^{2}(\Omega)}^{2}\right]+M e^{\gamma t_{0}}\right)\left\|v\left(t_{0}\right)\right\|_{L^{2}(\Omega)}^{2} \\
& +\epsilon M\left(e^{\frac{\gamma t}{2}}-e^{\frac{\gamma t_{0}}{2}}\right) \sup _{t \in\left[t_{0}, T\right]}\left[e^{\frac{\gamma t}{2}}\left\|\nu_{x}\right\|_{L^{2}(\Omega)}^{2}\right]+M\left(e^{\gamma t}-e^{\gamma t_{0}}\right) .
\end{aligned}
$$

Next, we multiply this inequality by $e^{-\frac{\gamma t}{2}}$, and by using (18) and (25), we obtain

$$
\begin{aligned}
e^{\frac{\gamma t}{2}}\left\|v_{x}\right\|_{L^{2}(\Omega)}^{2} \leq & e^{\gamma\left(t_{0}-\frac{t}{2}\right)} \Psi\left(v\left(t_{0}\right)\right)+M\left(e^{\frac{\gamma t}{2}}-e^{\gamma\left(t_{0}-\frac{t}{2}\right)}\right) \\
& +\frac{1}{2}\left(\epsilon e^{\frac{\gamma\left(t_{0}-t\right)}{2}} \sup _{t \in\left[t_{0}, T\right]}\left[e^{\frac{\gamma t}{2}}\left\|v_{x}\right\|_{L^{2}(\Omega)}^{2}\right]+M e^{\gamma\left(t_{0}-\frac{t}{2}\right)}\right)\left\|v\left(t_{0}\right)\right\|_{L^{2}(\Omega)}^{2} \\
& +\epsilon M\left(1-e^{\frac{\gamma\left(t_{0}-t\right)}{2}}\right) \sup _{t \in\left[t_{0}, T\right]}\left[e^{\frac{\gamma t}{2}}\left\|v_{x}\right\|_{L^{2}(\Omega)}^{2}\right] \\
\leq & e^{\gamma t_{0}} \Psi\left(v\left(t_{0}\right)\right)+\epsilon M \sup _{t \in\left[t_{0}, T\right]}\left[e^{\frac{\gamma t}{2}}\left\|v_{x}\right\|_{L^{2}(\Omega)}^{2}\right]+M e^{\frac{\gamma T}{2}}
\end{aligned}
$$


By taking the supremum on the left-hand side of the above inequality and by choosing $\epsilon=\frac{1}{2 M}$, we obtain

$$
\sup _{t \in\left[t_{0}, T\right]}\left[e^{\frac{\gamma t}{2}}\left\|\nu_{x}\right\|_{L^{2}(\Omega)}^{2}\right] \leq 2 e^{\gamma t_{0}} \Psi\left(v\left(t_{0}\right)\right)+M e^{\frac{\gamma T}{2}}
$$

and obviously,

$$
e^{\frac{\gamma T}{2}}\left\|v_{x}(T)\right\|_{L^{2}(\Omega)}^{2} \leq 2 e^{\gamma t_{0}} \Psi\left(\nu\left(t_{0}\right)\right)+M e^{\frac{\gamma T}{2}} .
$$

Since the time $T \geq 0$ is chosen arbitrarily, we can conclude that

$$
\left\|v_{x}(t)\right\|_{L^{2}(\Omega)}^{2} \leq 2 e^{\gamma\left(t_{0}-\frac{t}{2}\right)} \Psi\left(v\left(t_{0}\right)\right)+M \quad \text { for all } t \geq t_{0} .
$$

Finally, from the above inequality we can deduce that there exists $t^{\star} \geq t_{0}>0$ such that

$$
\|u(t)\|_{H^{1}(\Omega)}=\|v(t)\|_{H^{1}(\Omega)} \leq 2 M, \quad \forall t \geq t^{\star}
$$

In addition, from this established fact we can easily determine a closed subset $\mathcal{B}_{a b}$ which is a positive invariant for the semigroup $(S(t))_{t \geq 0}$.

\section{A splitting approach}

In this section, we present a splitting technical method in order to study the asymptotic compactness of the semigroup $(S(t))_{t \geq 0}$ in $\mathbf{L}^{2}(\boldsymbol{\Omega})$. For this procedure, the forcing term $f$ of NLS equation (2) is truncated as follows.

For $\alpha>0$, we consider a $\mathbf{C}^{\infty}$ cut-off function $\chi_{\alpha}$ defined by

$$
\chi_{\alpha}= \begin{cases}1 & \text { if }|x| \leq \alpha \\ 0 & \text { if }|x| \geq 1+\alpha\end{cases}
$$

such that $0 \leq \chi_{\alpha} \leq 1$. Since it is well known that the sequence $\left(f \chi_{\alpha}\right)_{\alpha}$ converges to $f$ in $\mathbf{L}^{2}(\boldsymbol{\Omega})$ as $\alpha \rightarrow+\infty$, then, for any $\left.\eta \in\right] 0,1[$, there exists $\alpha(\eta)$ such that

$$
\left\|f-f \chi_{\alpha(\eta)}\right\|_{\left.L^{(} \Omega\right)}^{2} \leq \eta
$$

By notation we set $f_{\eta}=f \chi_{\alpha(\eta)}$.

Next, we introduce a decomposition of the solution to NLS equation in order to prove the asymptotic compactness of the semigroup $(S(t))_{t \geq 0}$ in $\mathbf{L}^{2}(\boldsymbol{\Omega})$. Then, based on the above inequality, we decompose the solution $u$ of NLS equation (2)-(4)-(6) as a sum of two functions $u^{\eta}$ and $w^{\eta}$ such that

$$
\left\{\begin{array}{l}
i u_{t}^{\eta}+\frac{1}{2} u_{x x}^{\eta}+q \delta_{a} u^{\eta}+g\left(|u|^{2}\right) u^{\eta}+i \gamma u^{\eta}=f-f_{\eta} \quad \text { in } \Omega \times \mathbb{R}_{+}, \\
\partial_{n} u^{\eta}(0, t)+\sqrt{2} e^{-i \pi / 4} e^{-\gamma t} e^{i \mathbb{V}(0, t)} \partial_{t}^{1 / 2}\left(e^{\gamma t} e^{-i \mathbb{V}(0, t)} u^{\eta}(0, t)\right)=0, \quad t \in \mathbb{R}_{+}, \\
u^{\eta}(x, 0)=u_{0}(x), \quad x \in \Omega
\end{array}\right.
$$


and

$$
\left\{\begin{array}{l}
i w_{t}^{\eta}+\frac{1}{2} w_{x x}^{\eta}+q \delta_{a} w^{\eta}+g\left(|u|^{2}\right) w^{\eta}+i \gamma w^{\eta}=f_{\eta} \quad \text { in } \Omega \times \mathbb{R}_{+}, \\
\partial_{n} w^{\eta}(0, t)+\sqrt{2} e^{-i \pi / 4} e^{-\gamma t} e^{i \mathbb{V}(0, t)} \partial_{t}^{1 / 2}\left(e^{\gamma t} e^{-i \mathbb{V}(0, t)} w^{\eta}(0, t)\right)=0, \quad t \in \mathbb{R}_{+}, \\
w^{\eta}(x, 0)=0, \quad x \in \Omega,
\end{array}\right.
$$

where $\mathbb{V}(x, t)=\int_{0}^{t} g\left(|u(x, s)|^{2}\right) d s$.

In the sequel, we will show that the solution $u^{\eta}$ has a norm $\left\|u^{\eta}\right\|_{L^{2}(\Omega)}$ which can be chosen arbitrarily small. Also, we will show that the solution $w^{\eta}$ belongs to $\mathbf{H}^{\mathbf{1}}(\boldsymbol{\Omega}) \cap \mathbf{L}^{\mathbf{2}}(\boldsymbol{\Omega} ;(\mathbf{1}+$ $\left.\left.\mathbf{x}^{2}\right) \mathbf{d x}\right)$, where $\mathbf{L}^{2}\left(\boldsymbol{\Omega} ;\left(\mathbf{1}+\mathbf{x}^{2}\right) \mathbf{d x}\right)$ denotes the $L^{2}$ space endowed with the pondered norm $\|\star\|:=\left\|\star \sqrt{1+x^{2}}\right\|_{L^{2}(\Omega)}$.

We first study the long-time behavior of $u^{\eta}$ in the following technical result.

Lemma 3 Let $\mathcal{B}_{a b}$ be a bounded absorbing set of the semigroup $(S(t))_{t \geq 0}$. Then, for $u_{0} \in \mathcal{B}_{a b}$ and $\eta \in] 0,1[$, there exists $t(\eta)>0$ such that

$$
\forall t \geq 0, \quad\left\|u^{\eta}(t)\right\|_{L^{2}(\Omega)}^{2} \leq e^{-\gamma t}\left\|u_{0}\right\|_{L^{2}(\Omega)}^{2}+\frac{\eta}{\gamma^{2}}
$$

and

$$
\forall t \geq t(\eta), \quad\left\|u^{\eta}(t)\right\|_{L^{2}(\Omega)}^{2} \leq \frac{2 \eta}{\gamma^{2}}
$$

Proof Let $\tilde{u}^{\eta}(x, t)=\exp (-i \mathbb{V}(0, t)) u^{\eta}(x, t)$. Then equation (33) becomes

$$
\left\{\begin{array}{l}
i \tilde{u}_{t}^{\eta}+\frac{1}{2} \tilde{u}_{x x}^{\eta}+q \delta_{a} \tilde{u}^{\eta}+\left(g\left(|u(x, t)|^{2}\right)-g\left(|u(0, t)|^{2}\right)\right) \tilde{u}^{\eta}+i \gamma \tilde{u}^{\eta} \\
\quad=\exp (-i \mathbb{V}(0, t))\left(f-f_{\eta}\right) \quad \text { in } \Omega \times \mathbb{R}_{+}, \\
\partial_{n} \tilde{u}^{\eta}(0, t)+\sqrt{2} e^{-i \pi / 4} e^{-\gamma t} \partial_{t}^{1 / 2}\left(e^{\gamma t} \tilde{u}^{\eta}(0, t)\right)=0, \quad t \in \mathbb{R}_{+}, \\
\tilde{u}^{\eta}(x, 0)=u_{0}(x), \quad x \in \Omega .
\end{array}\right.
$$

Multiply equation (36) by $-i \overline{\tilde{u}^{\eta}}$, integrate in the space domain $\Omega$, and then integrate by parts the second term and consider the real part of the resulting equation

$$
\begin{aligned}
& \frac{1}{2} \frac{d}{d t}\left\|\tilde{u}^{\eta}(t)\right\|_{L^{2}(\Omega)}^{2}+\gamma\left\|\tilde{u}^{\eta}(t)\right\|_{L^{2}(\Omega)}^{2} \\
& \quad=\frac{1}{2} \mathcal{R} e\left(i \overline{\tilde{u}^{\eta}(0, t)} \partial_{n} \tilde{u}^{\eta}(0, t)\right)+\mathcal{I} m\left(\exp (-i \mathbb{V}(0, t)) \int_{-\infty}^{0}\left(f-f_{\eta}\right) \overline{\tilde{u}^{\eta}} d x\right) .
\end{aligned}
$$

Using the Cauchy-Schwarz and Young's inequalities, we get

$$
\begin{aligned}
& \frac{d}{d t}\left\|\tilde{u}^{\eta}(t)\right\|_{L^{2}(\Omega)}^{2}+\gamma\left\|\tilde{u}^{\eta}(t)\right\|_{L^{2}(\Omega)}^{2} \\
& \quad \leq \frac{1}{\gamma}\left\|f-f_{\eta}\right\|_{L^{2}(\Omega)}^{2}+\frac{1}{2} \mathcal{R} e\left(i \overline{\tilde{u}^{\eta}(0, t)} \partial_{n} \tilde{u}^{\eta}(0, t)\right) .
\end{aligned}
$$


We use Gronwall's lemma and (32) to obtain

$$
\begin{aligned}
\left\|\tilde{u}^{\eta}(t)\right\|_{L^{2}(\Omega)}^{2} \leq & e^{-\gamma t}\left\|u_{0}\right\|_{L^{2}(\Omega)}^{2}+\frac{1-e^{-\gamma t}}{\gamma^{2}} \eta \\
& +\frac{e^{-\gamma t}}{2} \int_{0}^{t} e^{\gamma s} \mathcal{R} e\left(i \overline{\tilde{u}^{\eta}(0, s)} \partial_{n} \tilde{u}^{\eta}(0, s)\right) d s .
\end{aligned}
$$

Using the boundary condition for NLS equation (36) and Lemma 1, we can show that

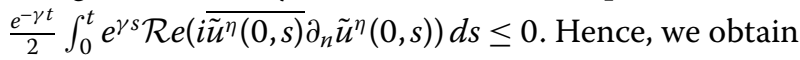

$$
\left\|u^{\eta}(t)\right\|_{L^{2}(\Omega)}^{2}=\left\|\tilde{u}^{\eta}(t)\right\|_{L^{2}(\Omega)}^{2} \leq e^{-\gamma t}\left\|u_{0}\right\|_{L^{2}(\Omega)}^{2}+\frac{\eta}{\gamma^{2}} .
$$

If $t \geq t(\eta)=\frac{1}{\gamma} \ln \left(\frac{\gamma^{2}\left\|u_{0}\right\|_{L^{2}(\Omega)}^{2}}{\eta}\right)>0$, we have

$$
\left\|u^{\eta}(t)\right\|_{L^{2}(\Omega)}^{2} \leq \frac{2 \eta}{\gamma^{2}} .
$$

Next, we show that $w^{\eta}$ remains bounded in $\mathbf{H}^{\mathbf{1}}(\boldsymbol{\Omega})$.

Lemma 4 There exists a constant $M$ depending on $\gamma, \alpha_{1}, \alpha_{2},\|f\|_{L^{2}(\Omega)}$ such that, for any $u_{0} \in \mathcal{B}_{a b}$, we have

$$
\forall t \geq 0, \quad\left\|w^{\eta}(t)\right\|_{H^{1}(\Omega)} \leq M
$$

Proof It follows from Theorem 2 and Lemma 3 that $w^{\eta}=u^{\eta}-u$ remains bounded in $\mathbf{L}^{2}(\boldsymbol{\Omega})$. To complete the proof, it suffices to show that the norm of the derivative of $w^{\eta},\left\|w_{x}^{\eta}\right\|_{L^{2}(\Omega)}$ is bounded.

For this aim, we set $\tilde{w}^{\eta}(x, t)=\exp (-i \mathbb{V}(0, t)) w^{\eta}(x, t)$. Then NLS equation (34) becomes

$$
\left\{\begin{array}{l}
i \tilde{w}_{t}^{\eta}+\frac{1}{2} \tilde{w}_{x x}^{\eta}+q \delta_{a} \tilde{w}^{\eta}+\left(g\left(|u(x, t)|^{2}\right)-g\left(|u(0, t)|^{2}\right)\right) \tilde{w}^{\eta}+i \gamma \tilde{w}^{\eta} \\
\quad=\exp (-i \mathbb{V}(0, t)) f_{\eta} \quad \text { in } \Omega \times \mathbb{R}_{+}, \\
\partial_{n} \tilde{w}^{\eta}(0, t)+\sqrt{2} e^{-i \pi / 4} e^{-\gamma t} \partial_{t}^{1 / 2}\left(e^{\gamma t} \tilde{w}^{\eta}(0, t)\right)=0, \quad t \in \mathbb{R}_{+}, \\
\tilde{w}^{\eta}(x, 0)=0, \quad x \in \Omega .
\end{array}\right.
$$

We multiply the first equality of (38) by $\overline{\tilde{w}_{t}^{\eta}+\gamma \tilde{w}^{\eta}}$ and integrate in the space domain $\Omega$. Then, by considering the real part, we get

$$
\begin{aligned}
\frac{d}{d t} \Phi & \left(\tilde{w}^{\eta}(t)\right)+2 \gamma \Phi\left(\tilde{w}^{\eta}(t)\right) \\
= & 2 \mathcal{R} e\left(\overline{\tilde{w}_{t}^{\eta}(0, t)+\gamma \tilde{w}^{\eta}(0, t)} \partial_{n} \tilde{w}^{\eta}(0, t)\right) \\
& +2 \int_{\Omega}\left(g\left(|u|^{2}\right)-g\left(|u(0, t)|^{2}\right)\right)\left(\frac{d}{d t}\left|\tilde{w}^{\eta}\right|^{2}+2 \gamma\left|\tilde{w}^{\eta}\right|^{2}\right) d x \\
& +4 \gamma \mathcal{R} e\left(\exp (-i \mathbb{V}(0, t)) \int_{-\infty}^{0} f_{\eta} \overline{\tilde{w}^{\eta}} d x\right) \\
& -4 \mathcal{R} e\left(i|u(0, t)|^{2} \exp (-i \mathbb{V}(0, t)) \int_{-\infty}^{0} f_{\eta} \overline{\tilde{w}^{\eta}} d x\right),
\end{aligned}
$$


where

$$
\begin{aligned}
\Phi\left(\tilde{w}^{\eta}(t)\right)= & \left\|\tilde{w}_{x}^{\eta}\right\|_{L^{2}(\Omega)}^{2}-2 q\left|\tilde{w}^{\eta}(a, t)\right|^{2} \\
& +4 \mathcal{R} e\left(\exp (-i \mathbb{V}(0, t)) \int_{-\infty}^{0} f_{\eta} \overline{\tilde{w}^{\eta}} d x\right) .
\end{aligned}
$$

Now, we need to majorize $\left\|\tilde{w}_{x}^{\eta}(t)\right\|_{L^{2}(\Omega)}^{2}$ in terms of $\Phi\left(\tilde{w}^{\eta}(t)\right)$. Indeed, we have by (40)

$$
\begin{aligned}
\left\|\tilde{w}_{x}^{\eta}\right\|_{L^{2}(\Omega)}^{2}= & \Phi\left(\tilde{w}^{\eta}(t)\right)+2 q\left|\tilde{w}^{\eta}(a, t)\right|^{2} \\
& -4 \mathcal{R} e\left(\exp (-i \mathbb{V}(0, t)) \int_{-\infty}^{0} f_{\eta} \overline{\tilde{w}^{\eta}} d x\right) .
\end{aligned}
$$

Note that from now on the quantity $M$ represents a constant depending on $\gamma, \alpha_{1}, \alpha_{2}$, $\|f\|_{L^{2}(\Omega)}$, which may vary from the context. By using Agmon's and Young's inequalities, we have

$$
2 q\left|\tilde{w}^{\eta}(a, t)\right|^{2} \leq 2|q|\left\|\tilde{w}^{\eta}\right\|_{L^{2}(\Omega)}\left\|\tilde{w}_{x}^{\eta}\right\|_{L^{2}(\Omega)} \leq \frac{1}{2}\left\|\tilde{w}_{x}^{\eta}\right\|_{L^{2}(\Omega)}^{2}+M
$$

Also, the last term of (41) can be bounded by using the Cauchy-Schwarz inequality as follows:

$$
-\mathcal{R} e\left(\exp (-i \mathbb{V}(0, t)) \int_{-\infty}^{0} f_{\eta} \overline{\tilde{w}^{\eta}} d x\right) \leq 4\left\|f_{\eta}\right\|_{L^{2}(\Omega)}\left\|\tilde{w}^{\eta}\right\|_{L^{2}(\Omega)} \leq M
$$

Hence, by using the above inequalities (42)-(43), we get

$$
\left\|\tilde{w}_{x}^{\eta}\right\|_{L^{2}(\Omega)}^{2} \leq 2 \Phi\left(\tilde{w}^{\eta}(t)\right)+M
$$

Next, we majorize the second member of equality (39).

\section{Majorization of the terms depending on $f_{\eta}$ :}

Since $u$ is bounded in $\mathbf{H}^{\mathbf{1}}(\boldsymbol{\Omega})$, then by using (43), the Cauchy-Schwarz inequality and Lemma 2, we have

$$
\begin{aligned}
& \left|\mathcal{R} e\left(i|u(0, t)|^{2} \exp (-i \mathbb{V}(0, t)) \int_{-\infty}^{0} f_{\eta} \overline{\tilde{w}^{\eta}} d x\right)\right| \\
& \quad \leq 2 M\|u\|_{L^{2}(\Omega)}\left\|u_{x}\right\|_{L^{2}(\Omega)} .
\end{aligned}
$$

By using (43), we deduce

$$
\begin{aligned}
& 4 \gamma \operatorname{Re}\left(\exp (-i \mathbb{V}(0, t)) \int_{-\infty}^{0} f_{\eta} \overline{\tilde{w}^{\eta}} d x\right) \\
& -4 \mathcal{R} e\left(i|u(0, t)|^{2} \exp (-i \mathbb{V}(0, t)) \int_{-\infty}^{0} f_{\eta} \overline{\tilde{w}^{\eta}} d x\right) \leq M .
\end{aligned}
$$

Majorization of the term $2 \int_{\Omega}\left(g\left(|u|^{2}\right)-g\left(|u(0, t)|^{2}\right)\right)\left(\frac{d}{d t}\left|\tilde{w}^{\eta}\right|^{2}+2 \gamma\left|\tilde{w}^{\eta}\right|^{2}\right) d x$ : 
We multiply the first equality of (38) by $\overline{\tilde{w}^{\eta}}$ and by considering the imaginary part, we get

$$
\frac{d}{d t}\left|\tilde{w}^{\eta}\right|^{2}+2 \gamma\left|\tilde{w}^{\eta}\right|^{2}=-2 \mathcal{I} m\left(\frac{d}{d x}\left(\tilde{w}_{x}^{\eta} \overline{\tilde{w}^{\eta}}\right)\right)+2 \mathcal{I} m\left(\exp (-i \mathbb{V}(0, t)) f \overline{\tilde{w}^{\eta}}\right)
$$

which implies

$$
\begin{aligned}
& 2 \int_{\Omega}\left(g\left(|u|^{2}\right)-g\left(|u(0, t)|^{2}\right)\right)\left(\frac{d}{d t}\left|\tilde{w}^{\eta}\right|^{2}+2 \gamma\left|\tilde{w}^{\eta}\right|^{2}\right) d x \\
& =-4 \int_{\Omega}\left(g\left(|u|^{2}\right)-g\left(|u(0, t)|^{2}\right)\right) \mathcal{I} m\left(\frac{d}{d x}\left(\tilde{w}_{x}^{\eta} \overline{\tilde{w}^{\eta}}\right)\right) d x \\
& \quad+4 \int_{\Omega}\left(g\left(|u|^{2}\right)-g\left(|u(0, t)|^{2}\right)\right) \mathcal{I} m\left(\exp (-i \mathbb{V}(0, t)) f \overline{\tilde{w}^{\eta}}\right) d x .
\end{aligned}
$$

By using integration by parts in $\Omega$, we get

$$
\begin{aligned}
& 2 \int_{\Omega}\left(g\left(|u|^{2}\right)-g\left(|u(0, t)|^{2}\right)\right)\left(\frac{d}{d t}\left|\tilde{w}^{\eta}\right|^{2}+2 \gamma\left|\tilde{w}^{\eta}\right|^{2}\right) d x \\
& =4 \int_{\Omega} \frac{d}{d x}\left(g\left(|u|^{2}\right)-g\left(|u(0, t)|^{2}\right)\right) \mathcal{I} m\left(\tilde{w}_{x}^{\eta} \overline{\tilde{w}^{\eta}}\right) d x \\
& \quad-4\left[\left(g\left(|u(x, t)|^{2}\right)-g\left(|u(0, t)|^{2}\right)\right) \mathcal{I} m\left(\tilde{w}_{x}^{\eta} \overline{\tilde{w}^{\eta}}\right)\right]_{-\infty}^{0} \\
& \quad+4 \int_{\Omega}\left(g\left(|u|^{2}\right)-g\left(|u(0, t)|^{2}\right)\right) \operatorname{Im}\left(\exp (-i \mathbb{V}(0, t)) f \overline{\tilde{w}^{\eta}}\right) d x .
\end{aligned}
$$

Since $-4\left[\left(g\left(|u(x, t)|^{2}\right)-g\left(|u(0, t)|^{2}\right)\right) \operatorname{I} m\left(\tilde{w}_{x}^{\eta} \overline{\tilde{w}^{\eta}}\right)\right]_{-\infty}^{0}=0$, we obtain

$$
\begin{aligned}
& 2 \int_{\Omega}\left(g\left(|u|^{2}\right)-g\left(|u(0, t)|^{2}\right)\right)\left(\frac{d}{d t}\left|\tilde{w}^{\eta}\right|^{2}+2 \gamma\left|\tilde{w}^{\eta}\right|^{2}\right) d x \\
& =8 \mathcal{R} e \int_{\Omega} u_{x} \bar{u} g^{\prime}\left(|u|^{2}\right) \mathcal{I} m\left(\tilde{w}_{x}^{\eta} \overline{\tilde{w}^{\eta}}\right) d x \\
& \quad+4 \int_{\Omega}\left(g\left(|u|^{2}\right)-g\left(|u(0, t)|^{2}\right)\right) \mathcal{I} m\left(\exp (-i \mathbb{V}(0, t)) f \overline{\tilde{w}^{\eta}}\right) d x .
\end{aligned}
$$

Taking into account assumption (3) on the function $g$, then by using Agmon's, Young's inequalities and inequality (44), the first term of the second member of (47) becomes

$$
\begin{aligned}
\left|8 \mathcal{R} e \int_{\Omega} u_{x} \bar{u} g^{\prime}\left(|u|^{2}\right) \mathcal{I} m\left(\tilde{w}_{x}^{\eta} \overline{\tilde{w}^{\eta}}\right) d x\right| & \leq 8 C\|u\|_{L^{\infty}(\Omega)}\left\|u_{x}\right\|_{L^{2}(\Omega)}\left\|\tilde{w}^{\eta}\right\|_{L^{\infty}(\Omega)}\left\|\tilde{w}_{x}^{\eta}\right\|_{L^{2}(\Omega)} \\
& \leq \gamma \Phi\left(\tilde{w}^{\eta}\right)+M .
\end{aligned}
$$

The second term of (47) can be shown to be bounded, by using (3), the Cauchy-Schwarz inequality and Lemma 2.

Since $u$ is bounded in $\mathbf{H}^{\mathbf{1}}(\boldsymbol{\Omega})$, we have

$$
\left|4 \int_{\Omega}\left(g\left(|u|^{2}\right)-g\left(|u(0, t)|^{2}\right)\right) \mathcal{I} m\left(\exp (-i \mathbb{V}(0, t)) f \overline{\tilde{w}^{\eta}}\right) d x\right| \leq M
$$


From the boundedness of the terms of (39), we deduce

$$
\frac{d}{d t} \Phi\left(\tilde{w}^{\eta}(t)\right)+\gamma \Phi\left(\tilde{w}^{\eta}(t)\right) \leq M+2 \mathcal{R} e\left(\overline{\tilde{w}_{t}^{\eta}(0, t)+\gamma \tilde{w}^{\eta}(0, t)} \partial_{n} \tilde{w}^{\eta}(0, t)\right)
$$

Finally, by applying Gronwall's lemma, Lemma 1 and inequality (44), we conclude that

$$
\forall t \geq 0, \quad\left\|w^{\eta}(t)\right\|_{H^{1}(\Omega)}=\left\|\tilde{w}^{\eta}(t)\right\|_{H^{1}(\Omega)} \leq 2 \Phi\left(\tilde{w}^{\eta}(t)\right)+M
$$

and hence $w^{\eta}$ is bounded in $\mathbf{H}^{\mathbf{1}}(\boldsymbol{\Omega})$.

Lemma 5 There exists a constant $M$ depending on $\gamma, \alpha_{1}, \alpha_{2},\|f\|_{L^{2}(\Omega)}$ such that for any $u_{0} \in \mathcal{B}_{a b}$, we have

$$
\forall t \geq 0, \quad\left\|x w^{\eta}(t)\right\|_{L^{2}(\Omega)} \leq M
$$

Proof We multiply equation (34) by $-i x^{2} \overline{w^{\eta}}$, we integrate in the space domain $\Omega$ and we use integration by parts of the real part of the resulting equation to obtain

$$
\begin{aligned}
& \frac{1}{2} \frac{d}{d t}\left\|x w^{\eta}(t)\right\|_{L^{2}(\Omega)}^{2}-\frac{1}{2} \mathcal{I} m\left[i x^{2} w_{x}^{\eta}(x, t) \overline{w^{\eta}(x, t)}\right]_{-\infty}^{0}+\gamma\left\|x w^{\eta}(t)\right\|_{L^{2}(\Omega)}^{2} \\
& \quad=\operatorname{Im}\left(\exp (-i \mathbb{V}(0, t)) \int_{-\infty}^{0} x^{2} f_{\eta} \overline{w^{\eta}} d x\right)+2 \mathcal{I} m\left(\int_{\Omega} x w_{x}^{\eta} \overline{w^{\eta}}\right)
\end{aligned}
$$

Using the fact that $\frac{1}{2} \mathcal{I} m\left[i x^{2} w_{x}^{\eta}(x, t) \overline{w^{\eta}(x, t)}\right]_{-\infty}^{0}=0$, the Cauchy-Schwarz and Young's inequalities, we get

$$
\frac{d}{d t}\left\|x w^{\eta}(t)\right\|_{L^{2}(\Omega)}^{2}+\gamma\left\|x w^{\eta}(t)\right\|_{L^{2}(\Omega)}^{2} \leq M
$$

Finally, the boundedness of the term $\left\|x w^{\eta}(t)\right\|_{L^{2}(\Omega)}$ results from Gronwall's lemma.

At this stage, we are in a place to show the compactness of the semigroup $(S(t))_{t \geq 0}$.

Proposition 3 For any $\epsilon>0$, there exists a time $t(\epsilon)>0$ such that, $\forall t \geq t(\epsilon), S(t) \mathcal{B}_{a b}$ may be covered by a finite number of balls of $\mathbf{L}^{2}(\boldsymbol{\Omega})$ of radius $\epsilon$; that is, there exists a finite sequence $x_{i} \in L^{2}(\Omega)$ such that

$$
S(t) \mathcal{B}_{a b} \subset \bigcup_{i} B_{L^{2}(\Omega)}\left(x_{i}, \epsilon\right)
$$

where $B_{L^{2}(\Omega)}\left(x_{i}, \epsilon\right)$ is the ball centered at $x_{i}$ with radius $\epsilon$.

Proof Let $\epsilon>0$ and choose $\eta=\min \left(1, \frac{\epsilon \gamma^{2}}{4}\right)$. We set $t(\epsilon)=t(\eta)$ as it was obtained by (35). Let $u_{0} \in \mathcal{B}_{a b}$ and recall that by the previous splitting procedure, we have

$$
S(t) u_{0}=u^{\eta}+w^{\eta} \quad \text { for } t \geq t(\epsilon)
$$


where $u^{\eta} \in B_{L^{2}(\Omega)}(0, \epsilon)$ and $w^{\eta}$ is bounded in $\mathbf{H}^{\mathbf{1}}(\boldsymbol{\Omega}) \cap \mathbf{L}^{2}\left(\boldsymbol{\Omega} ;\left(\mathbf{1}+\mathbf{x}^{\mathbf{2}}\right) \mathbf{d x}\right)$. Since the injection of $\mathbf{H}^{\mathbf{1}}(\boldsymbol{\Omega}) \cap \mathbf{L}^{\mathbf{2}}\left(\boldsymbol{\Omega} ;\left(\mathbf{1}+\mathbf{x}^{\mathbf{2}}\right) \mathbf{d x}\right)$ in $\mathbf{L}^{2}(\boldsymbol{\Omega})$ is compact, there exists a finite sequence $x_{i} \in L^{2}(\Omega)$ such that

$$
w^{\eta} \in \bigcup_{i} B_{L^{2}(\Omega)}\left(x_{i}, \frac{\epsilon}{2}\right),
$$

hence it holds $S(t) u_{0} \in \bigcup_{i} B_{L^{2}(\Omega)}\left(x_{i}, \epsilon\right)$.

\section{Maximal compact attractor}

The following result shows the precompactness of NLS trajectories in $\mathbf{L}^{2}(\boldsymbol{\Omega})$.

Proposition 4 Let $\left(u_{0}^{j}\right)_{j}$ be any sequence in $\mathcal{B}_{a b}$ and $\left(t_{j}\right)_{j}$ be any positive sequence such that $t_{j} \rightarrow+\infty$. Then $\left(S\left(t_{j}\right) u_{0}^{j}\right)_{j}$ is a relatively compact subset of $\mathbf{L}^{2}(\boldsymbol{\Omega})$.

Proof We show that $\left(S\left(t_{j}\right) u_{0}^{j}\right)_{j}$ has a subsequence which is strongly convergent in $\mathbf{L}^{2}(\boldsymbol{\Omega})$.

By Proposition 3 we have that for any $\epsilon>0$, there exists $t(\epsilon)>0$ such that if $t_{j}>t(\epsilon)$, then

$$
\left(S\left(t_{j}\right) u_{0}^{j}\right)_{j} \subset \bigcup_{h \in L^{2}(\Omega)} B_{L^{2}(\Omega)}(h, \epsilon) \subset \mathbf{L}^{2}(\boldsymbol{\Omega})
$$

where this union is finite. Then $\left(S\left(t_{j}\right) u_{0}^{j}\right)_{j}$ is precompact in $\mathbf{L}^{2}(\boldsymbol{\Omega})$. In addition, we prove that $\bigcup_{t \geq t(\epsilon)} S(t) \mathcal{B}_{a b}$ is closed in $\mathbf{L}^{2}(\boldsymbol{\Omega})$. Indeed, we consider a convergent sequence $\left(S\left(t_{j}\right) u_{0}^{j}\right)_{j}$ in $\bigcup_{t \geq t(\epsilon)} S(t) \mathcal{B}_{a b}$, and we show its convergence to an element $l$ in $\bigcup_{t \geq t(\epsilon)} S(t) \mathcal{B}_{a b}$.

By using the semigroup property, we have $S\left(t_{j}\right) u_{0}^{j}=S(t(\epsilon)) S\left(t_{j}-t(\epsilon)\right) u_{0}^{j}$. Also, by the positive invariance of $\mathcal{B}_{a b}$, it follows

$$
\left(S\left(t_{j}-t(\epsilon)\right) u_{0}^{j}\right)_{j} \subset \mathcal{B}_{a b}
$$

Then there exists a subsequence $\left(j_{k}\right)_{k}$ such that

$$
S\left(t_{j_{k}}-t(\epsilon)\right) u_{0}^{j_{k}} \rightarrow y \quad \text { in } \mathbf{H}^{\mathbf{1}}(\boldsymbol{\Omega}) .
$$

Finally, by using Proposition 2, we get

$$
S\left(t_{j_{k}}\right) u_{0}^{j_{k}}=S(t(\epsilon)) S\left(t_{j_{k}}-t(\epsilon)\right) u_{0}^{j_{k}} \rightarrow S(t(\epsilon)) y=l \in \bigcup_{t \geq t(\epsilon)} S(t) \mathcal{B}_{a b} .
$$

Now, we are able to announce our main result.

Theorem 3 The $\omega$-limit set $\mathcal{A}=w\left(\mathcal{B}_{a b}\right)=\bigcap_{s \geq 0} \overline{\bigcup_{t \geq s} S(t) \mathcal{B}_{a b}}$ of the bounded absorbing set $\mathcal{B}_{a b}$ (where the closure is taken with respect to the weak topology of $\mathbf{H}^{\mathbf{1}}(\boldsymbol{\Omega})$ ) is such that:

(i) $\mathcal{A}$ is bounded and weakly closed in $\mathbf{H}^{\mathbf{1}}(\boldsymbol{\Omega})$;

(ii) $S(t) \mathcal{A}=\mathcal{A}, \forall t \geq 0$;

(iii) for every bounded set $B$ in $\mathbf{H}^{\mathbf{1}}(\boldsymbol{\Omega})$,

$$
\operatorname{dist}_{H}(S(t) B, \mathcal{A}) \rightarrow 0 \quad \text { when } t \rightarrow+\infty,
$$

where dist $_{H}$ denotes the Hausdorff distance defined by $\operatorname{dist}_{H}(A, B)=\sup _{a \in A} \inf _{b \in B} d(a, b)$. 
Moreover, the set $\mathcal{A}$ is the global attractor of $(S(t))_{t \geq 0}$ in the weak topology of $\mathbf{H}^{\mathbf{1}}(\boldsymbol{\Omega})$.

The proof of Theorem 3 will be given further, and it is based on the following lemmas which show that $\mathcal{A}$ is invariant and attracts bounded sets of $\mathbf{H}^{\mathbf{1}}(\boldsymbol{\Omega})$.

Lemma 6 For all $t \geq 0$, it holds $\mathcal{A} \subset S(t) \mathcal{A}$.

Proof Let $b \in \mathcal{A}$, then by $\omega$-limit characterization, there exists a sequence $\left(t_{n}\right)_{n}$ of positive real numbers satisfying $t_{n} \rightarrow+\infty$ and a sequence $\left(b_{n}\right)_{n}$ of $\mathcal{B}_{a b}$ such that $S\left(t_{n}\right) b_{n} \rightarrow b$ weakly in $\mathbf{H}^{\mathbf{1}}(\boldsymbol{\Omega})$.

Let $t \geq 0$, since $t_{n}-t \rightarrow+\infty$ and $\left(S\left(t_{n}-t\right) b_{n}\right)_{n}$ is bounded in $\mathbf{H}^{\mathbf{1}}(\boldsymbol{\Omega})$. Then there exist subsequences $\left(t_{n_{k}}-t\right)_{k},\left(b_{n_{k}}\right)_{k} \subset \mathcal{B}_{a b}$ and an element $b^{\prime}$ such that $S\left(t_{n_{k}}-t\right) b_{n_{k}} \rightarrow b^{\prime}$ weakly in $\mathbf{H}^{\mathbf{1}}(\boldsymbol{\Omega})$ and $S\left(t_{n_{k}}-t\right) b_{n_{k}} \rightarrow b^{\prime}$ strongly in $\mathbf{L}^{2}(\boldsymbol{\Omega})$. Then $b^{\prime} \in \mathcal{A}$. By using Proposition 2 it follows $S\left(t_{n_{k}}\right) b_{n_{k}} \rightarrow S(t) b^{\prime}$ in $\mathbf{L}^{2}(\boldsymbol{\Omega})$. Hence, we conclude by uniqueness of the limit that $b=S(t) b^{\prime} \in S(t) \mathcal{A}$.

Lemma 7 For all $t \geq t^{\prime}$, it holds $S\left(t^{\prime}\right) \mathcal{A} \subset S(t) \mathcal{A}$.

Proof Let $\psi \in S\left(t^{\prime}\right) \mathcal{A}$, then there exists $\phi \in \mathcal{A}$ such that $\psi=S\left(t^{\prime}\right) \phi$. Since $t-t^{\prime} \geq 0$, then by Lemma 6, we have $\phi \in S\left(t-t^{\prime}\right) \mathcal{A}$. We can deduce that there exists $\tilde{\phi} \in \mathcal{A}$ such that $\phi=S\left(t-t^{\prime}\right) \tilde{\phi}$, which implies

$$
\psi=S\left(t^{\prime}\right) S\left(t-t^{\prime}\right) \tilde{\phi}=S(t) \tilde{\phi} \in S(t) \mathcal{A}
$$

Lemma 8 For all $t \geq 0$, it holds $S(t) \mathcal{A} \subset \mathcal{A}$.

Proof We prove the result by contradiction. Hence, we assume that $\exists t_{0}>0, \exists b \in \mathcal{A}$ such that $S\left(t_{0}\right) b \notin \mathcal{A}$. Using Lemma 7, we have $S\left(t_{0}\right) b \in S\left(t_{0}\right) \mathcal{A} \subset S(t) \mathcal{A}, \forall t \geq t_{0}$. Then we can construct a sequence $\left(b_{n}\right)_{n}$ of $\mathcal{A}$ associated with a positive time sequence $\left(t_{n}\right)_{n}$ such that $t_{n} \rightarrow+\infty$ satisfying $S\left(t_{n}\right) b_{n}=S\left(t_{0}\right) b$. As $\left(S\left(t_{n}\right) b_{n}\right)$ is bounded in $\mathbf{H}^{\mathbf{1}}(\boldsymbol{\Omega})$, then there exist a subsequence $\left(t_{n_{k}}\right)_{k}$, a subsequence $\left(b_{n_{k}}\right)_{k} \subset \mathcal{A}$ and $e \in \mathbf{H}^{\mathbf{1}}(\boldsymbol{\Omega})$ such that $S\left(t_{n_{k}}\right) b_{n_{k}} \rightarrow e$ weakly in $\mathbf{H}^{\mathbf{1}}(\boldsymbol{\Omega})$. This implies $e \in \omega(\mathcal{A}) \subset \mathcal{A}$, which leads to a contradiction since $S\left(t_{0}\right) b=$ $e \in \mathcal{A}$ by the fact that the sequence $S\left(t_{n_{k}}\right) b_{n_{k}}$ is stationary.

Hence, we have shown that $\forall t \geq 0, S(t) \mathcal{A} \subset \mathcal{A}$.

Next, we show that $\mathcal{A}$ attracts the bounded set of $\mathbf{H}^{\mathbf{1}}(\boldsymbol{\Omega})$.

Lemma $9 \mathcal{A}$ is attractive, i.e., $\operatorname{dist}_{H}(S(t) B, \mathcal{A}) \rightarrow 0$ when $t \rightarrow+\infty$, for any bounded set $B$ of $H^{1}(\Omega)$.

Proof We prove the result by contradiction.

Let $B$ be a bounded set $B$ of $H^{1}(\Omega)$. We assume that there exist $\delta>0$, a sequence $\left(t_{n}\right)_{n}$ of positive real numbers such that $t_{n} \rightarrow+\infty$ and a sequence $\left(b_{n}\right)_{n}$ in $B$ such that $d\left(S\left(t_{n}\right) b_{n}, \mathcal{A}\right) \geq \delta>0$ satisfying

$$
S\left(t_{n}\right) b_{n}=S\left(t_{n}-t(B)\right) S(t(B)) b_{n}
$$


where $t(B)$ is the entry time in the bounded absorbent $\mathcal{B}_{a b}$. As $t_{n}-t(B) \rightarrow+\infty$ and $S\left(t_{n}-\right.$ $t(B)) S(t(B)) b_{n}$ is bounded in $\mathbf{H}^{\mathbf{1}}(\boldsymbol{\Omega})$, then there exist a subsequence $\left(t_{n_{k}}\right)_{k}$ of positive real numbers such that $t_{n_{k}} \rightarrow+\infty$, a subsequence $\left(S(t(B)) b_{n_{k}}\right)_{k} \subset \mathcal{B}_{a b}$ and $b \in \mathbf{H}^{\mathbf{1}}(\boldsymbol{\Omega})$ such that

$$
S\left(t_{n_{k}}-t(B)\right) S(t(B)) b_{n_{k}} \rightarrow b \quad \text { weakly in } \mathbf{H}^{\mathbf{1}}(\boldsymbol{\Omega})
$$

which implies $b \in \mathcal{A}$ and $d\left(S\left(t_{n_{k}}\right) b_{n_{k}}, \mathcal{A}\right) \geq \delta>0$. This leads to a contradiction.

Proof of Theorem 3 Note that condition (i) is trivial since we use the weak topology of $\mathbf{H}^{\mathbf{1}}(\boldsymbol{\Omega})$.

Condition (ii) is an immediate consequence of Lemmas 6, 7 and 8.

Finally, condition (iii) holds from the result of Lemma 9.

\section{Conclusion}

In this paper, we have studied a nonlinear Schrödinger equation with Dirac interaction defect. For this purpose, artificial boundary conditions were used in order to characterize the behavior of the solutions. Moreover, we have shown that the introduced Schrödinger equation can be characterized by an autonomous dynamical system. In addition, we have proved the existence of a maximal compact attractor in the weak topology of $\mathbf{H}^{\mathbf{1}}(\boldsymbol{\Omega})$.

The remaining issue is to investigate the NLS equation on a bounded domain with artificial boundary conditions. This is a delicate problem which needs future investigation.

\footnotetext{
Acknowledgements

The authors are greatly indebted to Professor Olivier Goubet from Picardie Jules Verne university, France, and Professor Mustapha Ait Rami from EMSI Marrakesh, Morocco, for their helpful comments and suggestions.
}

Competing interests

The authors declare that they have no competing interests.

\section{Authors' contributions}

All authors contributed equally to the writing of this paper. All authors read and approved the final manuscript.

\section{Publisher's Note}

Springer Nature remains neutral with regard to jurisdictional claims in published maps and institutional affiliations.

Received: 26 December 2016 Accepted: 1 May 2017 Published online: 12 May 2017

\section{References}

1. Gross, EP: Structure of a quantized vortex in boson systems. Nuovo Cimento 20,454-477 (1961)

2. PitaevskiĬ, LP: Vortex lines in an imperfect Bose gas. Sov. Phys. JETP 13, 451-454 (1961)

3. Goodman, RH, Holmes, PJ, Weinstein, MI: Strong NLS soliton-defect interactions. Physica D 192, $215-248$ (2004)

4. Holmer, J, Zworski, M: Slow soliton interaction with delta impurities. arXiv:math/0702465 (2007)

5. Sulem, C, Sulem, P-L: The Nonlinear Schrödinger Equation: Self-Focusing and Wave Collapse. Applied Mathematical Sciences, vol. 139. Springer, New York (1999)

6. Burgnies, L, Vanbésien, O, Lippens, D: Transient analysis of ballistic transport in stublike quantum waveguides. Appl. Phys. Lett. 71, 803-805 (1997)

7. Levy, M: Parabolic Equation Methods for Electromagnetic Wave Propagation. IEE Electromagnetic Waves Series, vol. 45. Institution of Electrical Engineers, London (2000)

8. Claerbout, JF: Coarse grid calculation of waves in inhomogeneous media with application to delineation of complicated seismic structure. Geophysics 35, 407-418 (1970)

9. Schmidt, F, Deuflhard, P: Discrete transparent boundary conditions for the numerical solution of Fresnel's equation. Comput. Math. Appl. 29(9), 53-76 (1995)

10. Tappert, FD: The Parabolic Approximation Method, pp. 224-287. Springer, Berlin (1977)

11. Gazol, A, Passot, T, Sulem, PL: Coupling between nonlinear Alfvén waves and reduced magnetohydrodynamics for compressible fluids. Phys. Plasmas 6(8), 3114-3122 (1999)

12. Holmer, J, Marzuola, J, Zworski, M: Fast soliton scattering by delta impurities. Commun. Math. Phys. 274(1), 187-216 (2007) 
13. Ghidaglia, J-M: Finite dimensional behavior for weakly damped driven Schrödinger equations. Ann. Inst. Henri Poincaré, Anal. Non Linéaire 5(4), 365-405 (1988)

14. Laurençot, $P$ : Long-time behaviour for weakly damped driven nonlinear Schrödinger equations in $\mathbf{R}^{N}, N \leq 3$. NoDEA Nonlinear Differ. Equ. Appl. 2, 357-369 (1995)

15. Goubet, O: Regularity of the attractor for a weakly damped nonlinear Schrödinger equation. Appl. Anal. 60(1-2), 99-119 (1996)

16. Kechiche, W: Systèmes d'équations de Schrödinger non linéaires. PhD thesis, Université de Monastir (2012)

17. Antoine, X, Arnold, A, Besse, C, Ehrhardt, M, Schädle, A: A review of transparent and artificial boundary conditions techniques for linear and nonlinear Schrödinger equations. Commun. Comput. Phys. 4 729-796 (2008)

18. Antoine, $X$, Besse, $C$, Klein, $P$ : Absorbing boundary conditions for Schrödinger equations with general potentials and nonlinearities. SIAM J. Sci. Comput. 33, 1008-1033 (2011)

19. Antoine, X, Besse, C, Klein, P: Absorbing boundary conditions for the one-dimensional Schrödinger equation with an exterior repulsive potential. J. Comput. Phys. 228, 312-335 (2009)

20. Chrifi, A: Analyse des schémas numériques et comportement asymptotique de certaines edp dispersives. PhD thesis, Cadi Ayyad University (in preparation)

21. Abounouh, M, Al Moatassime, H, Chrifi, A: Galerkin method of weakly damped cubic nonlinear Schrödinger with Dirac impurity, and artificial boundary condition in a half-space. Appl. Comput. Math. (2017)

22. Arnold, A: Numerically absorbing boundary conditions for quantum evolution equations. VLSI Des. 6, 313-319 (1998)

\section{Submit your manuscript to a SpringerOpen ${ }^{\circ}$ journal and benefit from:}

- Convenient online submission

Rigorous peer review

- Immediate publication on acceptance

- Open access: articles freely available online

- High visibility within the field

- Retaining the copyright to your article 